\title{
Genome-Wide Identification and Functional Characterization of CCHC-Type Zinc Finger Genes in Ustilaginoidea virens
}

\author{
Xiaoyang Chen ${ }^{1,2} \mathbb{1}$, Zhangxin Pei ${ }^{3}$, Lin Peng ${ }^{1,2}$, Qin Qin ${ }^{1,2}$, Yuhang Duan ${ }^{1,2}$, Hao Liu ${ }^{1}$, Xiaolin Chen ${ }^{1,2} \mathbb{D}^{\text {, }}$ \\ Lu Zheng ${ }^{1,2}$, Chaoxi Luo ${ }^{1}$ and Junbin Huang ${ }^{1, *}$ \\ 1 Hubei Key Laboratory of Plant Pathology, Huazhong Agricultural University, Wuhan 430070, China; \\ chenxiaoyang2345@163.com (X.C.); Plin18943606383@163.com (L.P.); hzauqinqin980223@163.com (Q.Q.); \\ duan199607272021@163.com (Y.D.); hl210@mail.hzau.edu.cn (H.L.); chenxiaolin@mail.hzau.edu.cn (X.C.); \\ luzheng@mail.hzau.edu.cn (L.Z.); cxluo@mail.hzau.edu.cn (C.L.) \\ 2 State Key Laboratory of Agricultural Microbiology, Huazhong Agricultural University, Wuhan 430070, China \\ 3 Wuhan Institute of Landscape Architecture, Wuhan 430081, China; peizhangxinhzau@163.com \\ * Correspondence: junbinhuang@mail.hzau.edu.cn; Tel.: +86-150-7145-9466
}

\section{check for}

updates

Citation: Chen, X.; Pei, Z.; Peng, L.; Qin, Q.; Duan, Y.; Liu, H.; Chen, X.;

Zheng, L.; Luo, C.; Huang, J.

Genome-Wide Identification and

Functional Characterization of

CCHC-Type Zinc Finger Genes in

Ustilaginoidea virens. J. Fungi 2021, 7,

947. https://doi.org/10.3390/

jof7110947

Academic Editor: Katrina

Maria Ramonell

Received: 6 October 2021

Accepted: 27 October 2021

Published: 10 November 2021

Publisher's Note: MDPI stays neutral with regard to jurisdictional claims in published maps and institutional affiliations.

Copyright: (c) 2021 by the authors. Licensee MDPI, Basel, Switzerland. This article is an open access article distributed under the terms and conditions of the Creative Commons Attribution (CC BY) license (https:// creativecommons.org/licenses/by/ $4.0 /)$.

\begin{abstract}
Rice false smut caused by Ustilaginoidea virens is a serious disease of rice (Oryza sativa), severely reducing plant mass and yields worldwide. We performed genome-wide analysis of the CCHC-type zinc-finger transcription factor family in this pathogen. We identified and functionally characterized seven $U v C C H C$ genes in $U$. virens. The deletion of various $U v C C H C$ genes affected the stress responses, vegetative growth, conidiation, and virulence of $U$. virens. $\triangle U v C C H C 5$ mutants infected rice spikelets normally but could not form smut balls. Sugar utilization experiments showed that the $\triangle U v C C H C 5$ mutants were defective in the utilization of glucose, sucrose, lactose, stachyose, and trehalose. Deletion of UvCCHC5 did not affect the expression of rice genes associated with grain filling, as revealed by RT-qPCR. We propose that the $\triangle U v C C H C 5$ mutants are impaired in transmembrane transport, and the resulting nutrient deficiencies prevent them from using nutrients from rice to form smut balls. RNA-seq data analysis indicated that $U v C C H C 4$ affects the expression of genes involved in mitochondrial biogenesis, ribosomes, transporters, and ribosome biogenesis. These findings improve our understanding of the molecular mechanism underlying smut ball formation in rice by $U$. virens.
\end{abstract}

Keywords: rice false smut; Ustilaginoidea virens; CCHC-type zinc finger proteins; development; virulence

\section{Introduction}

Transcription factors are essential players in the development and virulence of plant pathogens that directly or indirectly regulate the expression of downstream genes [1]. The transcription factor families involved in phytopathogen development and virulence include bZIP (basic leucine zipper), bHLH (basic helix-loop-helix), homeodomain-like, MYB, MADS-box, APSES, and zinc-finger proteins [2]. The zinc-finger protein family is a large and diverse superfamily of proteins. Zinc-finger proteins use finger-like structures containing $\mathrm{Zn}^{2+}$ to bind DNA or RNA nucleotides and recognize DNA-RNA complexes or other proteins [3], thereby regulating the transcription or translation of genes that function in crucial processes such as growth, apoptosis, cell proliferation, and differentiation. The zinc finger is a tetrahedral structure formed by cysteine (Cys) and histidine (His) combined with zinc ions. Zinc-finger proteins are divided into different types based on their number and arrangement of $\mathrm{Cys}$ and His residues, such as $\mathrm{C} 2 \mathrm{HC}, \mathrm{C} 2 \mathrm{H} 2, \mathrm{C} 3 \mathrm{HC} 4, \mathrm{C} 2 \mathrm{HC} 5$, and so on [4], as well as CCHC-type zinc finger proteins.

CCHC-type zinc-finger proteins are highly conserved and widely distributed in animals, plants, and microorganisms [5]. All CCHC-type zinc-finger proteins contain the conserved CCHC-box domain "-C-X2-C-X4-H-X4-C-". The number or spatial structure of the $\mathrm{CCHC}$ box determines the type of motif or protein that it can recognize. In general, 
zinc-finger proteins containing one $\mathrm{CCHC}$ box bind to single-stranded DNA or RNA. Zinc-finger proteins containing three $\mathrm{CCHC}$ boxes are better able to bind to single-stranded RNA, including mRNA or miRNA in eukaryotic cells [6,7]. In plants, CCHC-type zincfinger proteins play key roles in regulating growth, development, and both abiotic and biotic stress responses [8-19]. In animals, these proteins are involved in regulating DNA recognition, RNA processing, transcriptional activation, cell apoptosis, and so on [20-22]. The yeast genome encodes at least seven CCHC-type zinc-finger proteins including Sf1, Slu7, Mpe1, Air1, Air2, Gis2, and Bik1; six of these are involved in RNA metabolism [5]. Sf1 is involved in the assembly of early spliceosome complexes. Slu7 is required for splicing fidelity $[23,24]$. Mpe1 is required for pre-mRNA cleavage and for polyadenylation at the bona fide site [25]. Air1 and Air2 are RNA-binding proteins [5]. Gis2 is considered a translational activator since it promotes cap-independent translation through interactions with the $5^{\prime}$-terminal oligopyrimidine tract of mRNAs and the translating ribosome [26]. Bik1 plays critical roles in the regulation of microtubule polymerization, stabilization, and dynamics [27]. However, the roles of CCHC-type zinc-finger proteins in fungal pathogens are still unknown.

Rice false smut caused by Ustilaginoidea virens, a devastating grain disease of rice, has become a problem in most rice-growing areas worldwide [28]. In addition to causing yield losses, rice false smut also threatens human and animal health by producing cyclopeptide mycotoxins in smut balls $[29,30]$. Comparative genomic analysis of $U$. virens and other filamentous ascomycetes predicted that $U$. virens possesses over 300 transcription factors [31,32], but only a few have thus far been characterized. For example, UvPRO1 plays key roles in the stress responses, vegetative growth, conidiation, and virulence of $U$. virens [33]. UvHox2 regulates the conidiation, chlamydospore formation, and virulence of $U$. virens [34]. UvCom 1 regulates the conidiation, stress responses, vegetative growth, and virulence of $U$. virens [35]. The $\mathrm{Zn}(2)-\mathrm{Cys}(6)$ class fungus-specific transcription factor UvZnFTF1 is involved in the conidiation, vegetative growth, pigment biosynthesis, and virulence of $U$. virens [36]. The $\mathrm{C} 2 \mathrm{H} 2$-type zinc-finger transcription factor UvMSN2 plays a key role in the conidiation, stress responses, vegetative growth, mitochondrial morphology, and virulence of $U$. virens [37]. The C2H2-type zinc-finger transcription factor UvCGBP1 also plays important roles in the conidiation, stress responses, vegetative growth, and virulence of $U$. virens. Finally, UvCGBP1 regulates the translation and transcription of the MAPK pathway kinase gene UvSlt2, which plays an important role in virulence [38]. Nonetheless, the roles of most transcription factors in $U$. virens are unknown.

In this study, we performed genome-wide identification and functional characterization of CCHC-type zinc-finger proteins in $U$. virens. Our findings demonstrate that CCHC-type zinc-finger proteins play important roles in regulating smut ball formation, conidiation, stress responses, vegetative growth, and virulence in $U$. virens.

\section{Materials and Methods}

\subsection{Fungal Strains and Growth Conditions}

The $U$. virens wild-type strain HWD-2 and all transformed strains were cultured on potato sucrose agar (PSA) medium at $28^{\circ} \mathrm{C}$ in the dark. Seven-day-old mycelia cultured in potato sucrose broth (PSB) shaken at $180 \mathrm{rpm}$ were used to isolate fungal DNA/RNA, and conidia separated from the culture were used for Agrobacterium tumefaciens-mediated transformation (ATMT) as described by Chen et al. [39].

\subsection{Transactivation Activity Assay in Yeast}

The transcriptional activities of UvCCHC proteins were examined by yeast one-hybrid assays using the Matchmaker pGBKT7 (Clontech, Mountain View, CA, USA) system. The coding sequences of $U v C C H C$ genes were ligated into the pGBKT7 vector for expression under the control of the GAL4 promoter. The resulting pGBKT7-UvCCHC vectors were transformed into Y1HGold (Clontech, Mountain View, CA, USA) cells, and transformants were isolated in SD/-Trp medium and confirmed by PCR with primer pair P1/P2. The 
transcriptional activities of the UvCCHC proteins were examined based on yeast growth on SD/-Trp/-His medium with X- $\alpha$-Gal (Clontech, Mountain View, CA, USA).

\subsection{DNA/RNA Manipulation and RT-qPCR}

Genomic DNA was extracted from vegetative hyphae using the CTAB method. Total RNA was extracted from vegetative hyphae and infected rice spikelets $(1,3,5,7,9$, or 13 days) using an RNA kit (Thermo Fisher Scientific, Waltham, MA, USA). cDNA synthesis was performed using cDNA Synthesis SuperMix (TransGen Biotech, Beijing, China). Quantitative reverse-transcription PCR (RT-qPCR) was conducted using TransStart ${ }^{\circledR}$ Tip Green qPCR SuperMix (TransGen Biotech, Beijing, China) to detect the expression levels of UvCCHC or rice grain filling-associated genes at different stages of infection with primer pairs (Supplementary Table S1); the $U$. virens $\beta$-tubulin (Uv8b_900) or rice ubiquitin gene (OsUBQ1), respectively, were used as internal controls [40].

\subsection{Gene Deletion and Complementation}

Deletion mutants of UvCCHC genes were generated using ATMT. Approximately $1000 \mathrm{bp}$ of the downstream and upstream flanking sequences of the genes were ligated into the pGKO deletion vector. The pGKO-UvCCHC constructs were inserted into A. tumefaciens strain EHA105 cells, which were used to transform HWD-2 conidia. Hygromycin-resistant transformants were isolated and confirmed by PCR. For the complementation assays, an approximately $5-\mathrm{kb}$ fragment containing a $1.5-\mathrm{kb}$ native promoter region and the fulllength UvCCHC gene sequence were amplified with primer pair P7/P8, and the resulting PCR products were ligated into pNeo3300III. A. tumefaciens strain EHA105 harboring the pNeo3300III-UvCCHC constructs were transformed by ATMT by co-culturing the agrobacteria with conidia of the $\triangle U v C C H C$ mutants. G418-resistant transformants (those carrying constructs) were isolated and confirmed by PCR with primer pairs P1/P2, P3/P4, P3/P6 and P5/P6.

\subsection{Vegetative Growth and Conidiation}

For vegetative growth, 5-mm mycelial plugs were obtained from 10-d-old PSA plates and grown on fresh PSA medium at $28^{\circ} \mathrm{C}$. After $14 \mathrm{~d}$ of incubation, the radial growth of vegetative mycelia was measured. For conidial production, strains were grown in PSB medium at $28^{\circ} \mathrm{C}$. After shaking at $180 \mathrm{rpm}$ for $7 \mathrm{~d}$, the cultures were filtered through four layers of gauze, and conidial production was measured using a hemocytometer. Each treatment was repeated three times [39].

\subsection{Stress Adaptation Assays and Sugar Utilization Assays}

To test the sensitivity of the cultures to environmental stress, $5-\mathrm{mm}$ mycelial plugs were cultured on PSA plates containing $0.25 \mathrm{M} \mathrm{NaCl}, 0.5 \mathrm{M}$ sorbitol, $0.02 \%$ or $0.04 \% \mathrm{H}_{2} \mathrm{O}_{2}$, $0.03 \%$ sodium dodecyl sulfate (SDS), $120 \mu \mathrm{g} / \mathrm{mL}$ Calcofluor white (CFW), or $120 \mu \mathrm{g} / \mathrm{mL}$ Congo red (CR). For the sugar utilization assays, after $10 \mathrm{~d}$ of growth on PSA plates, 5-mm mycelial plugs of HWD-2 and the mutated strains $\triangle U v C C H C 5-23, \triangle U v C C H C 5-43$, and $\mathrm{C} \triangle U_{v} \mathrm{CCHC5}-23$ were transferred to Czapek-Dox Agar medium $\left(2 \mathrm{~g} \mathrm{KNO}_{3}, 1 \mathrm{~g} \mathrm{~K} \mathrm{HPO}_{4}\right.$, $0.01 \mathrm{~g} \mathrm{FeSO}_{4}, 0.5 \mathrm{~g} \mathrm{KCl}, 0.5 \mathrm{~g} \mathrm{MgSO} 4 \cdot 7 \mathrm{H}_{2} \mathrm{O}, 16 \mathrm{~g}$ agar, $30 \mathrm{~g}$ sugar sources, with ddH ${ }_{2} \mathrm{O}$ to a total volume of $1000 \mathrm{~mL}$ ) with different sugar sources, including glucose, sucrose, lactose, maltose, raffinose, stachyose, trehalose, and soluble starch. After $14 \mathrm{~d}$ of incubation, the radial growth of vegetative mycelia was measured; three replicates were grown per strain.

\subsection{Pathogenicity and Plant Infection Assays}

The susceptible rice cultivar Wanxian-98 was used for pathogenicity assays [36]. The wild-type HWD-2, $\triangle U v C C H C$ mutant, and complementation strains were cultured in PSB medium at $28{ }^{\circ} \mathrm{C}$ for $7 \mathrm{~d}$ with shaking at $180 \mathrm{rpm}$. Rice plants were inoculated with $2 \mathrm{~mL}$ mycelial/spore suspensions $\left(1 \times 10^{6}\right.$ conidia/mL) at the booting stage using a syringe. The inoculated rice plants were cultivated in a greenhouse at $25^{\circ} \mathrm{C}$ with a relative humidity of 
$100 \%$ for $7 \mathrm{~d}$, and then grown in a greenhouse at $28^{\circ} \mathrm{C}$ with a relative humidity of $90 \%$ for a further $14 \mathrm{~d}$. The number of smut balls was then counted. The inoculation experiment was repeated three times, and each strain was inoculated onto 15 panicles each time. Rice spikelet samples were collected at three days post-inoculation (dpi) and $5 \mathrm{dpi}$ in $2.5 \%$ glutaraldehyde fixative. The samples were critical-point dried, mounted on stubs, sputter coated with gold-palladium, and viewed under a JEOL JSM-6390LV scanning electron microscope (SEM) operating at $10 \mathrm{kV}$.

\subsection{Generation of the GFP-UvCCHC Fusion Construct}

The full-length UvCCHC4 or UvCCHC5 coding sequence was ligated into the pNeo3300III-

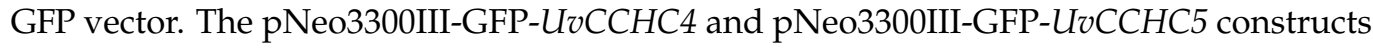
were inserted into $A$. tumefaciens strain EHA105 cells, which were used to transform $\triangle U v C C H C 4-1$ and $\triangle U v C C H C 5-23$ conidia, respectively. The transformants were verified by PCR with primer pair P1/P2 and immunoblotting. GFP-tagged strains were chosen to observe the subcellular localizations of the resulting fusion proteins under a Zeiss LSM510 Meta confocal microscope (Zeiss, Jena, Germany).

\subsection{RNA-seq and Data Analysis}

Total RNA extracted at 3 dpi from three biological replicates of rice spikelets infected with the HWD-2 and $\triangle U v C C H C 4-1$ strains was used for RNA sequencing (RNA-seq). RNA-seq libraries were constructed using an NEBNext ${ }^{\circledR}$ Ultra $^{\mathrm{TM}}$ Directional RNA Library Prep Kit using $5 \mu \mathrm{g}$ total RNA. RNA was first isolated using the polyA selection method by oligo(dT) beads and then fragmented by fragmentation buffer. Double-stranded cDNA was then synthesized using a SuperScript double-stranded cDNA Synthesis Kit (Invitrogen, Carlsbad, CA, USA). Then the synthesized cDNA was subjected to end-repair, phosphorylation and 'A' base addition according to Illumina's library construction protocol. Libraries were size-selected for cDNA target fragments of 200-300 bp on 2\% Low Range Ultra Agarose followed by PCR. After quantification by TBS380, paired-end RNA-seq libraries were sequenced on an Illumina HiSeq 4000 system with 150-bp paired-end reads at IGENEBOOK Biotechnology Co., Ltd. (Wuhan, China). For RNA-seq data analysis, the raw data of paired-end reads were filtered using SeqPrep and Sickle with default parameters. Cufflinks (version 2.2.1) and TopHat (version 2.0.14) software were used to map clean reads to the $U$. virens genome and to calculate differential expression. The expression level of each transcript was calculated as fragments per kilobase of exon per million mapped reads (FRKM). RSEM software (http:/ / deweylab.biostat.wisc.edu/rsem/, accessed on 24 May 2021) was used to quantify gene abundances [41]. The filter conditions of fold change $>2$ and adjusted $p<0.05$ were applied to identify differentially expressed genes. Gene Ontology (GO) functional enrichment and Kyoto Encyclopedia of Genes and Genomes (KEGG) pathway analysis were carried out by Goatools (https:/ / github.com/tanghaibao/Goatools, accessed on 20 July 2021) and KOBAS (http:/ / kobas.cbi.pku.edu.cn/home.do, accessed on 22 July 2021) [42].

\subsection{Accession Numbers}

The accession numbers of the rice grain filling-associated genes mentioned in this article are: OsSSIIIa (LOC_Os08g09230); OsRISBZ1 (LOC_Os07g08420); OsBEIIb (LOC_Os02g32660); OsAGPS2b (LOC_Os08g25734); OsAGPL2 (LOC_Os01g44220); OsPromln2 (LOC_Os05g26770); OsSSI (LOC_Os06g06560); OsGlutln3 (LOC_Os02g15090); OsUBQ1 (LOC_Os03g13170).

\subsection{Statistical Analysis}

The data were statistically analyzed using SPSS version 16.0 software (SPSS Inc., Chicago, IL, USA) and are presented as the mean \pm standard deviation (SD). 


\section{Results}

\subsection{Identification of CCHC-Type Zinc-Finger Proteins in $U$. virens}

We identified seven CCHC-type zinc-finger genes in the $U$. virens genome based on the presence of the conserved CCHC-box domain "-C-X2-C-X4-H-X4-C-". Phylogenetic analysis of CCHC-type zinc-finger protein homologs from different fungi revealed that these proteins are conserved in filamentous fungi (Figure 1A). We identified seven genes encoding proteins containing one to seven copies of the conserved $\mathrm{CCHC}$ box, suggesting that seven CCHC-type zinc-finger proteins are present in this fungus (Figure 1B; Table 1). To explore the transcriptional activity of these proteins, we performed a yeast one-hybrid assay by transforming Y1HGold cells with pGBKT7-UvCCHC constructs harboring the seven putative $\mathrm{Uv} v \mathrm{CCHC}$ genes. Transformants harboring $\mathrm{UvCCHC}_{v}, \mathrm{UvCCHC4}, \mathrm{UvCCHC}$, or $U v C C H C 7$ grew normally and appeared blue on $\mathrm{SD} /-\operatorname{Trp} /-$ His plates, revealing transactivation activity in yeast (Figure 1C). These results suggest that UvCCHC3, UvCCHC4, UvCCHC5, and UvCCHC7 function as transcription factors in $U$. virens.

A

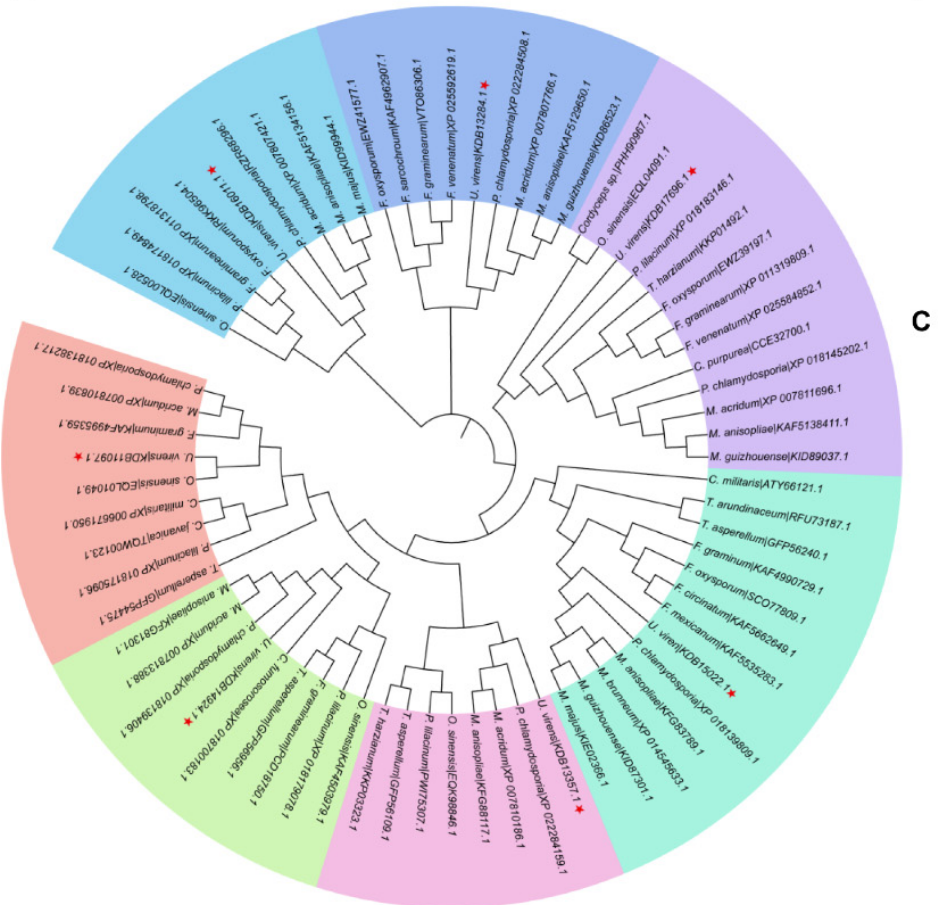

B

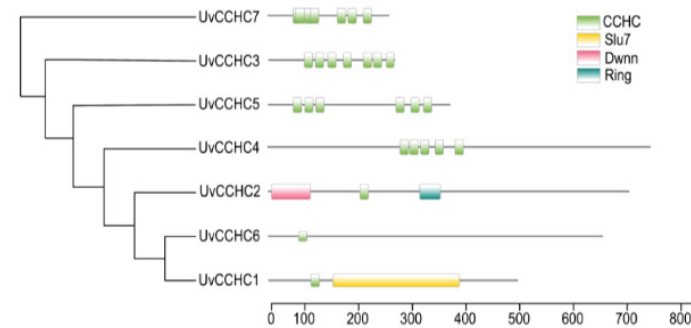

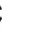

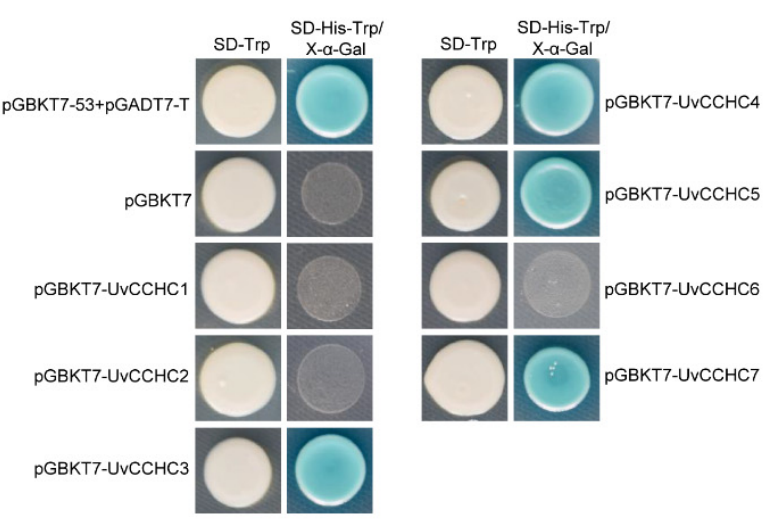

Figure 1. Identification of CCHC-type zinc-finger proteins in $U$. virens. (A) Neighbor-joining tree of CCHC-type zincfinger protein homologs from different fungal genomes generated with MEGA7.0. The bootstrap percentage values from 1000 repeats are shown at the branch nodes. (B) Predicted Pfam domains of CCHC-type zinc-finger (box) proteins. (C) Transactivation analysis of CCHC-type zinc-finger proteins in yeast. The vectors pGBKT7-53/pGADT7-T and pGBKT7 were expressed in yeast as positive and negative controls, respectively.

\subsection{Expression Patterns of CCHC-Type Zinc-Finger Genes in U. virens}

To further investigate the functions of the UvCCHC transcription factors, we examined the expression profiles of these $U v C C H C$ genes in $U$. virens at various stages of infection through RT-qPCR with primer pair P9/P10. Compared to the vegetative-mycelial stage (0 dpi), UvPal1 was highly expressed during mycelial expansion to form smut balls (7-13 dpi) in rice spikelets, as a control [39], and the expression levels of UvCCHC genes significantly increased during the early stage of hypha infection in rice floral organs at 3-5 dpi (Figure 2). These observations suggest that $U v C C H C$ genes play important roles during early infection of $U$. virens. 
Table 1. Genome-wide identification of CCHC-type zinc-finger genes in U. virens.

\begin{tabular}{ccc}
\hline Gene ID & Function Description & Name \\
\hline Uv8b_1580 & Pre-mRNA-splicing factor slu7 & UvCCHC1 \\
Uv8b_2941 & Retinoblastoma-binding protein & UvCCHC2 \\
Uv8b_4070 & Cellular nucleic acid-binding protein & UvCCHC3 \\
Uv8b_4168 & Zinc-knuckle domain-containing protein & UvCCHC4 \\
Uv8b_5736 & Putative zinc-knuckle transcription factor & UvCCHC5 \\
Uv8b_5874 & Zinc-finger domain-containing protein & UvCCHC6 \\
Uv8b_8085 & Zinc-knuckle domain-containing protein & UvCCHC7 \\
\hline
\end{tabular}
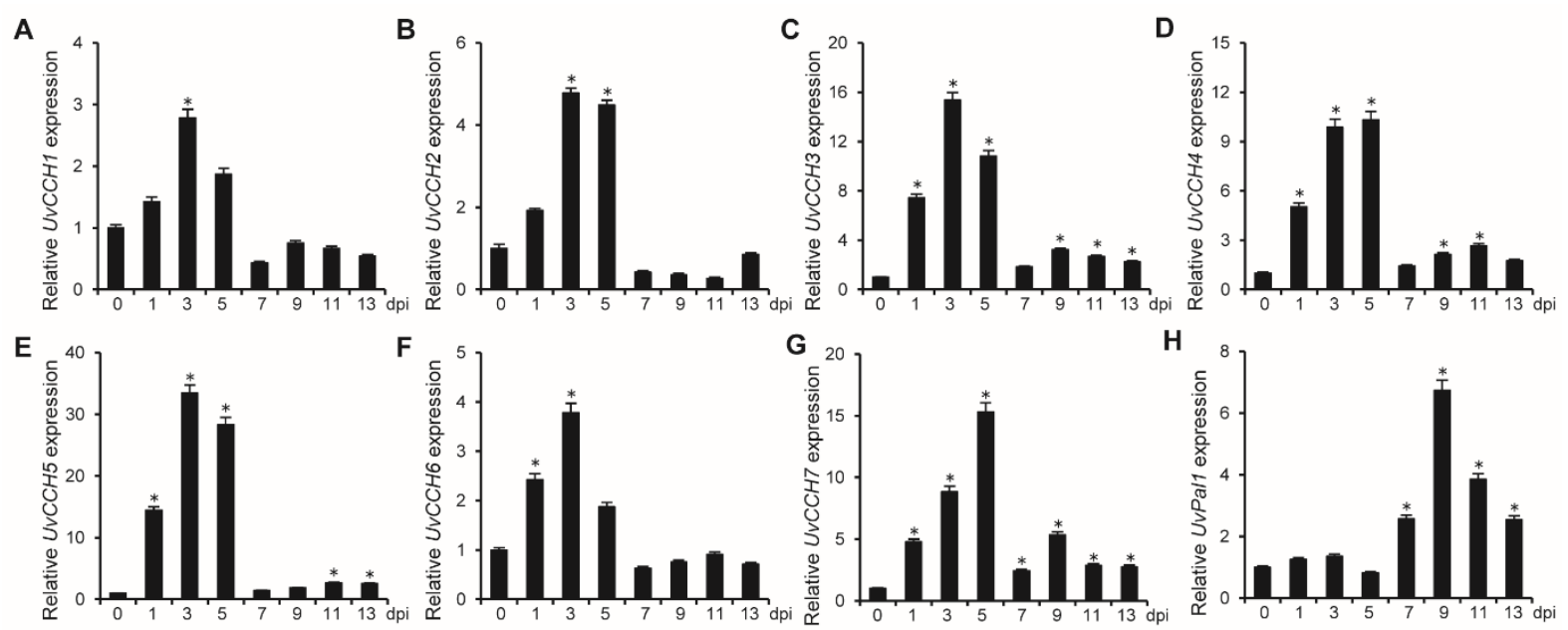

Figure 2. Expression of $U v C C H C$ genes in $U$. virens. (A-H) Expression profiles of $U v C C H C$ and $U v$ Pal1 genes relative to $\beta$-tubulin in hyphae ( $0 \mathrm{dpi}$ ) in PSB and at different stages of infection on rice (1-13 d), as determined by RT-qPCR. Asterisks represent significant differences in comparison with 0 dpi at $P=0.05$.

\subsection{UvCCHC Genes Are Important for the Mycelial Growth and Conidiation of $U$. virens}

We generated $U v C C H C$ gene knockout mutants by deleting the $U v C C H C$ genes using a homologous recombination strategy, as confirmed by PCR analysis (Supplementary Figure S1; Table 2). To investigate whether $\mathrm{UvCCHC}$ genes are associated with the mycelial growth of $U$. virens, we measured the growth rates of wild-type strain HWD-2, the $\triangle U v C C H C$ mutants, and the complementation strains on PSA medium. Compared to that of HWD-2, the mycelial growth rates of the $\triangle U v C C H C 3$ and $\triangle U v C C H C 4$ mutants were significantly reduced, whereas those of the $\triangle U v C C H C 1$ mutants were significantly increased (Figure $3 \mathrm{~A}, \mathrm{~B}$ ). We also assessed conidiation in all $U$. virens strains when incubated in PSB with shaking. Conidial production was significantly reduced in the $\triangle U v C C H 5$ mutant but significantly increased in the $\triangle U v C C H 6$ and $\triangle U v C C H 7$ mutants compared to wild-type HWD-2

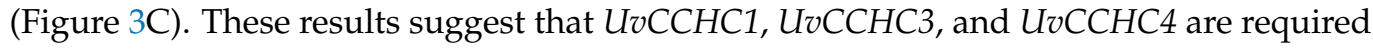

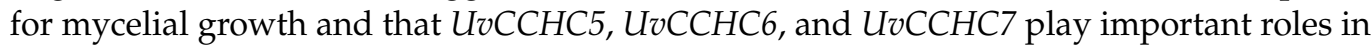
the conidiation of $U$. virens.

\subsection{UvCCHC Genes Play Key Roles in the Responses of U. virens to Environmental Stress}

To explore the roles of $U v C C H C$ genes in mediating the adaptation of $U$. virens to environmental stress, we compared the radial growth rates of HWD-2, the $\triangle U v C C H C$ mutants, and the complementation strains on PSA containing different stress agents. These chemicals significantly inhibited the mycelial growth of the $\triangle U v C C H C$ mutants compared to HWD-2 (Figure 4; Supplementary Figure S2). These results indicate that $U v C C H C$ genes regulate the responses of $U$. virens to osmotic stress and oxidative stress, as well as its cell wall integrity. 
Table 2. Wild-type and mutant strains of $U$. virens used in this study.

\begin{tabular}{ccc}
\hline Strain & Description & Reference \\
\hline HWD-2 & Wild-type strain & {$[35]$} \\
$\Delta U v C C H C 1-5$ & $U v C C H C 1$ deletion mutant of HWD-2 & This study \\
$\Delta U v C C H C 1-73$ & $U v C C H C 1$ deletion mutant of HWD-2 & This study \\
C $\Delta U v C C H C 1-5$ & $U v C C H C 1$ complementation strain from $\triangle U v C C H C 1-5$ & This study \\
$\Delta U v C C H C 2-36$ & $U v C C H C 2$ deletion mutant of HWD-2 & This study \\
$\Delta U v C C H C 2-51$ & $U v C C H C 2$ deletion mutant of HWD-2 & This study \\
C $\Delta U v C C H C 2-36$ & $U v C C H C 3$ complementation strain from $\triangle U v C C H C 2-36$ & This study \\
$\Delta U v C C H C 3-16$ & $U v C C H C 3$ deletion mutant of HWD-2 & This study \\
$\Delta U v C C H C 3-37$ & $U v C C H C 3$ deletion mutant of HWD-2 & This study \\
C $\triangle U v C C H C 3-16$ & $U v C C H C 3$ complementation strain from $\triangle U v C C H C 3-16$ & This study \\
$\Delta U v C C H C 4-1$ & $U v C C H C 4$ deletion mutant of HWD-2 & This study \\
$\Delta U v C C H C 4-11$ & $U v C C H C 4$ deletion mutant of HWD-2 & This study \\
C $\triangle U v C C H C 4-1$ & $U v C C H C 4$ complementation strain from $\triangle U v C C H C 4-1$ & This study \\
$\Delta U v C C H C 5-23$ & $U v C C H C 5$ deletion mutant of HWD-2 & This study \\
$\Delta U v C C H C 5-43$ & $U v C C H C 5$ deletion mutant of HWD-2 & This study \\
C $\triangle U v C C H C 5-23$ & $U v C C H C 5$ complementation strain from $\triangle U v C C H C 5-23$ & This study \\
$\Delta U v C C H C 6-8$ & $U v C C H C 6$ deletion mutant of HWD-2 & This study \\
$\Delta U v C C H C 6-22$ & $U v C C H C 6$ deletion mutant of HWD-2 & This study \\
C $\triangle U v C C H C 6-8$ & $U v C C H C 6$ complementation strain from $\triangle U v C C H C 6-8$ & This study \\
$\Delta U v C C H C 7-18$ & $U v C C H C 7$ deletion mutant of HWD-2 & This study \\
$\Delta U v C C H C 7-63$ & $U v C C H C 7$ deletion mutant of HWD-2 & This study \\
C $\Delta U v C C H C 7-18$ & $U v C C H C 7$ complementation strain from $\triangle U v C C H C 7-18$ & This study \\
\hline
\end{tabular}

\subsection{UvCCHC Genes Play Crucial Roles in the Virulence of $U$. virens}

To explore the roles of the $U v C C H C$ genes in $U$. virens infection, we conducted virulence assays of HWD-2, the $\triangle U v C C H C$ mutants, and the complementation strains on the susceptible rice cultivar Wanxian-98. After $21 \mathrm{dpi}$, we observed approximately 62 smut balls on rice spikelets infected by HWD-2 and the complementation strains, and approximately 60 smut balls on rice spikelets infected by $\triangle U v C C H C 1, \triangle U v C C H C 2$, and $\triangle U v C C H C 3$. The pathogenicity of $\triangle U v C C H C 6$ and $\triangle U v C C H C 7$ was significantly higher, as rice spikelets infected by these mutants produced more than 90 smut balls. By contrast, the virulence of the $\triangle U v C C H C 4$ and $\triangle U v C C H C 5$ mutants was significantly reduced, as $\triangle U v C C H C 4$ produced only approximately 24 smut balls on rice spikelets (Figure 5), and the $\triangle U v C C H C 5$ mutants failed to produce smut balls. These results indicate that UvCCHC6 and UvCCHC7 negatively regulate the virulence of $U$. virens, $U v C C H C 4$ and $U v C C H C 5$ positively regulate the virulence of this pathogen, and $U v C C H C 1, U v C C H C 2$, and $U v C C H C 3$ have no effect on its virulence.

\subsection{UvCCHC5 Regulates the Formation of Smut Balls}

Although the $\triangle U v C C H C 5$ mutants failed to produce smut balls, these mutants were able to infect rice floral organs. Therefore, we observed the infection process of HWD-2 and $\triangle U v C C H C 5-23$ in more detail (Figure 6A). In both strains, at 3 dpi, hyphae elongated and extended along the surfaces of spikelets. At $5 \mathrm{dpi}$, hyphae were observed on the surfaces of filaments in colonized rice. At $15 \mathrm{dpi}$, rice grains infected by $\triangle U v C C H C 5-23 \mathrm{did}$ not contain ball-like colonies, whereas infection by HWD-2 led to the formation of smut balls (Figure 6A). These results indicate that $\triangle U v C C H C 5-23$ successfully established a nutritional relationship with rice spikelets but could not form smut balls. 
A

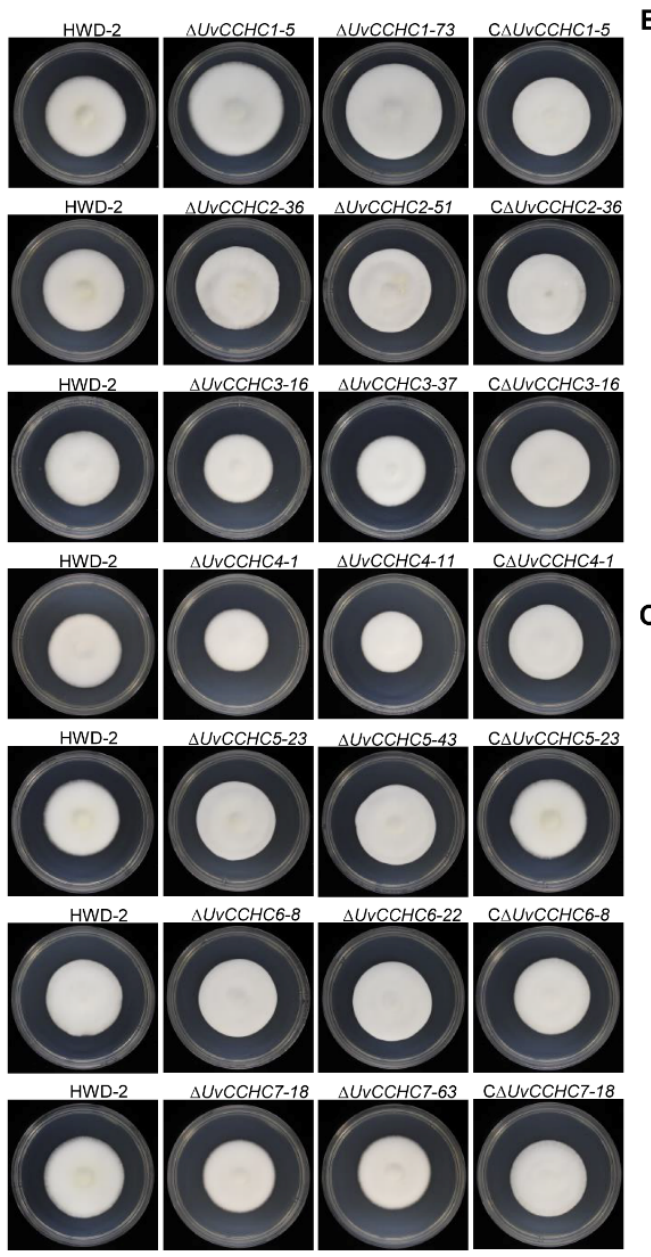

B

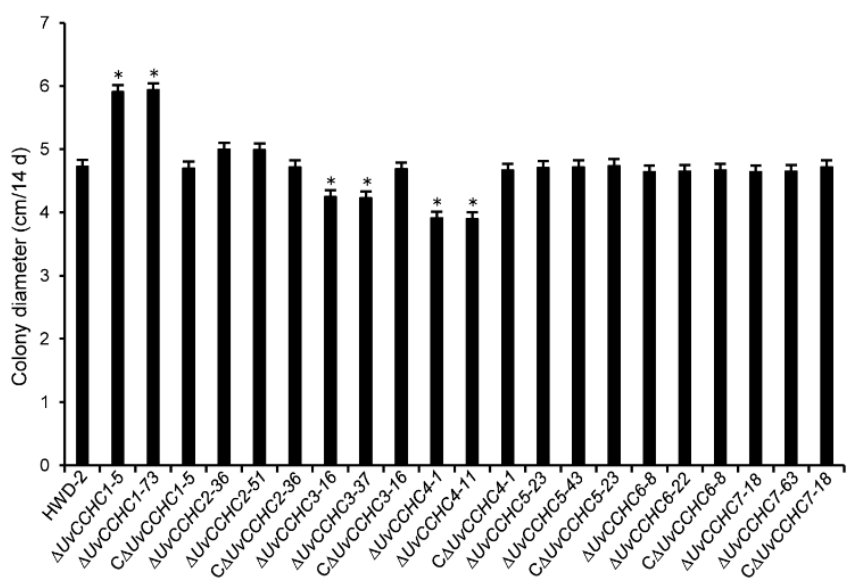

C

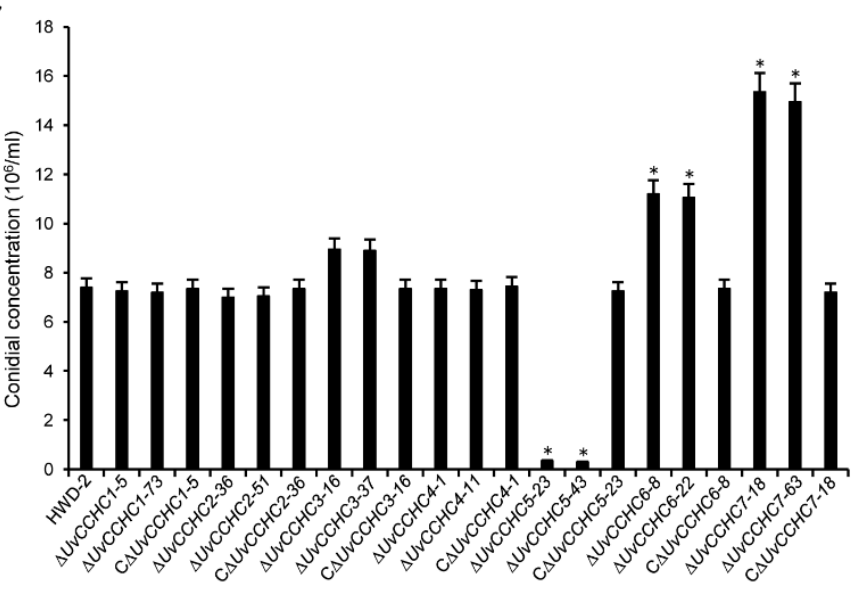

Figure 3. Deletion of $U v C C H C$ genes affects hyphal growth and conidiation. (A) Colony morphology of HWD-2, $\triangle U v C C H C$ mutants, and complementation strains grown on PSA for $14 \mathrm{~d}$. (B) Colony diameter of the mutant strains grown on PSA for $14 \mathrm{~d}$. (C) Conidial production of the mutant strains incubated in PSB at $180 \mathrm{rpm}$ for $7 \mathrm{~d}$. Asterisks represent significant differences between HWD-2 and the mutants at $p=0.05$, as determined by least significant difference (LSD) test.

\subsection{Deletion of UvCCHC5 Does Not Affect the Expression of Rice Genes Associated with Grain Filling}

$U$. virens infection in rice induces the expression of genes associated with grain filling by simulating fertilization, causing the rice to provide large amounts of nutrients to the pathogen $[43,44]$. We reasoned that unlike wild-type HWD-2, the $\triangle U v C C H C 5-23$ mutant cannot induce the expression of grain-filling genes and therefore cannot obtain large amounts of nutrients, preventing the formation of smut balls. To explore this hypothesis, we measured the expression of genes associated with grain filling in rice after inoculation with $\triangle U v C C H C 5-23$ or HWD-2 at different stages of infection. The expression levels of rice grain filling-associated genes, including OsSSIIIa, OsRISBZ1, OsBEIIb, OsAGPS2b, OsSSI, OsPromln2, OsAGPL2, and OsGlutln3, were similar in rice spikelets inoculated with the $\triangle U v C C H C 5-23$ mutant and HWD-2 (Figure 6B-I). These results indicate that the deletion of $\mathrm{UvCCHC} 5$ did not affect the expression of rice genes associated with grain filling. Therefore, the failure of $\triangle U v C C H C 5-23$ to induce smut ball formation is likely due to a deficiency in pathogen development. 
A

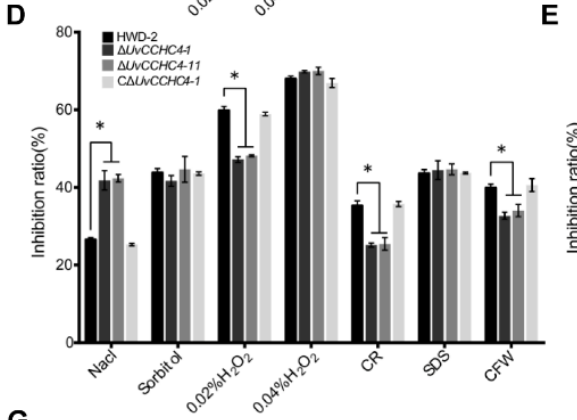

G
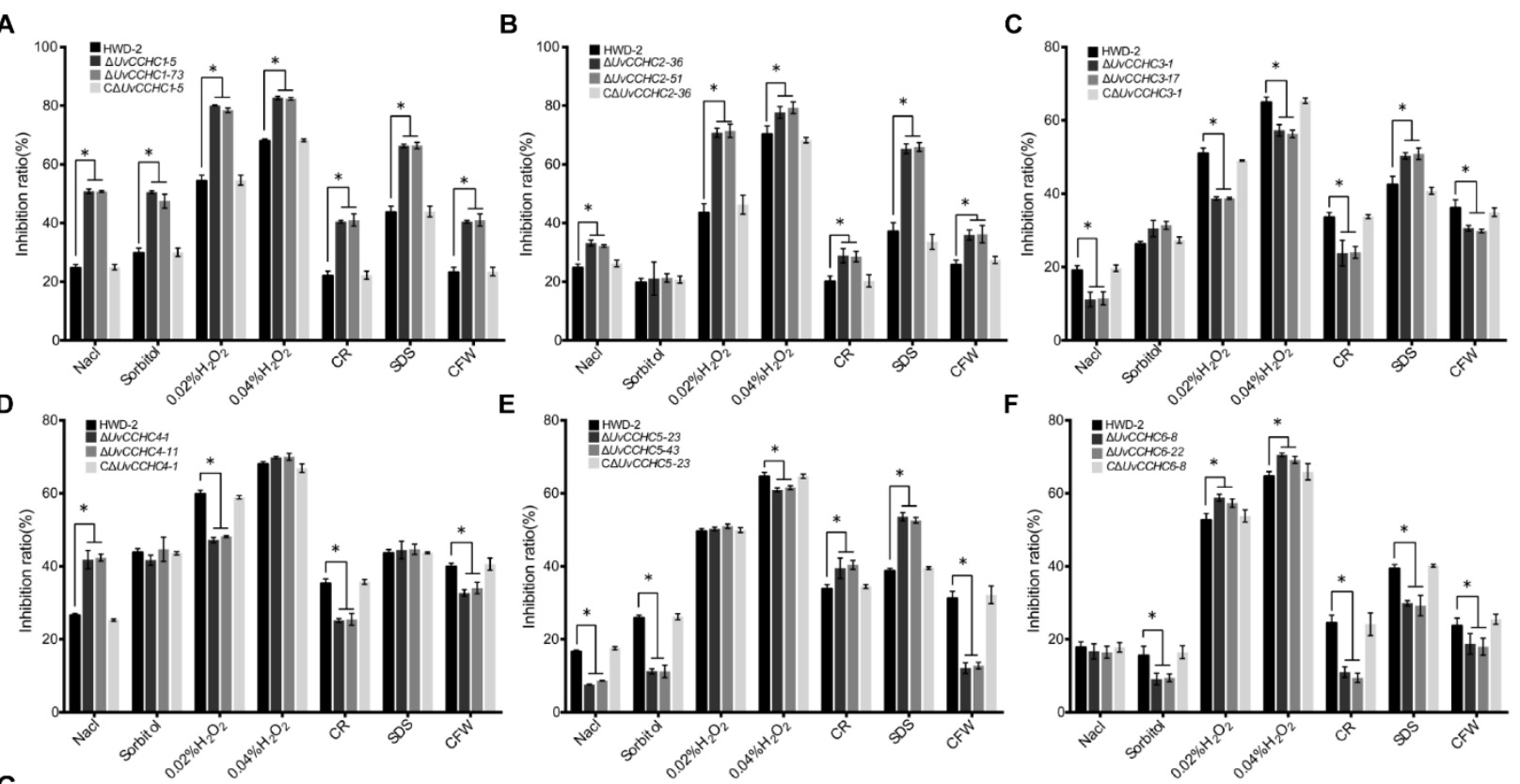

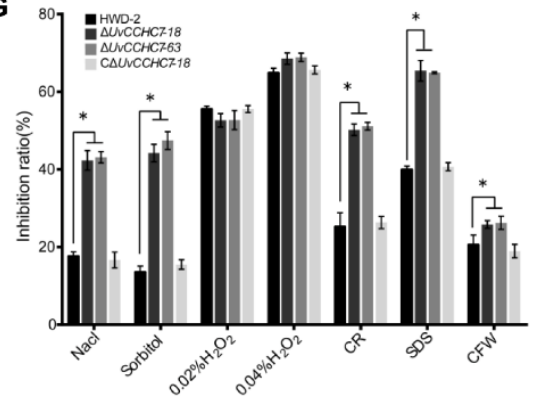

Figure 4. The $\triangle U v C C H C$ mutants are sensitive to various stresses. (A-G) Inhibition of colony growth of the mutant strains on PSA supplied with $0.25 \mathrm{M} \mathrm{NaCl}, 0.5 \mathrm{M}$ sorbitol, $0.02 \%$ or $0.04 \% \mathrm{H}_{2} \mathrm{O}_{2}, 0.03 \% \mathrm{SDS}, 0.12 \mathrm{mg} / \mathrm{mL} \mathrm{CR}$, or $0.12 \mathrm{mg} / \mathrm{mL} \mathrm{CFW}$ after $14 \mathrm{~d}$ of incubation at $28{ }^{\circ} \mathrm{C}$. Asterisks represent significant differences between HWD-2 and the mutants at $p=0.05$, as determined by LSD test.

\subsection{UvCCHC5 Plays Important Roles in Sugar Utilization, Carbohydrate Transport, and Transmembrane Transport}

Like wild-type $U$. virens, $\triangle U v C C H C 5-23$ induced the expression of genes associated with grain filling in rice spikelets. We reasoned that the failure of infected spikelets to form smut balls was due not to the lack of a nutrient supply but instead to the impaired use of nutrients by $U$. virens after $U v C C H C 5$ gene knockout. Therefore, we compared the carbohydrate utilization preferences of $\triangle U v C C H C 5-23$ and $\triangle U v C C H C 5-43$ with those of HWD-2 and the complementation strain C $\triangle U v C C H C 5-23$. We grew 5-mm mycelial plugs from these strains on Czapek-Dox Agar medium containing different saccharides, including sucrose, lactose, glucose, maltose, stachyose, raffinose, trehalose, and soluble starch. Compared to HWD-2 and CAUvCCHC5-23, the growth rates of the $\triangle U v C C H C 5$ mutants were significantly reduced on glucose, sucrose, lactose, stachyose, and trehalose media, indicating that the mutant had defects in the utilization of these saccharides (Figure 7A,B). These results suggest that UvCCHC5 is involved in the utilization of glucose, sucrose, lactose, stachyose, and trehalose by $U$. virens. 
A
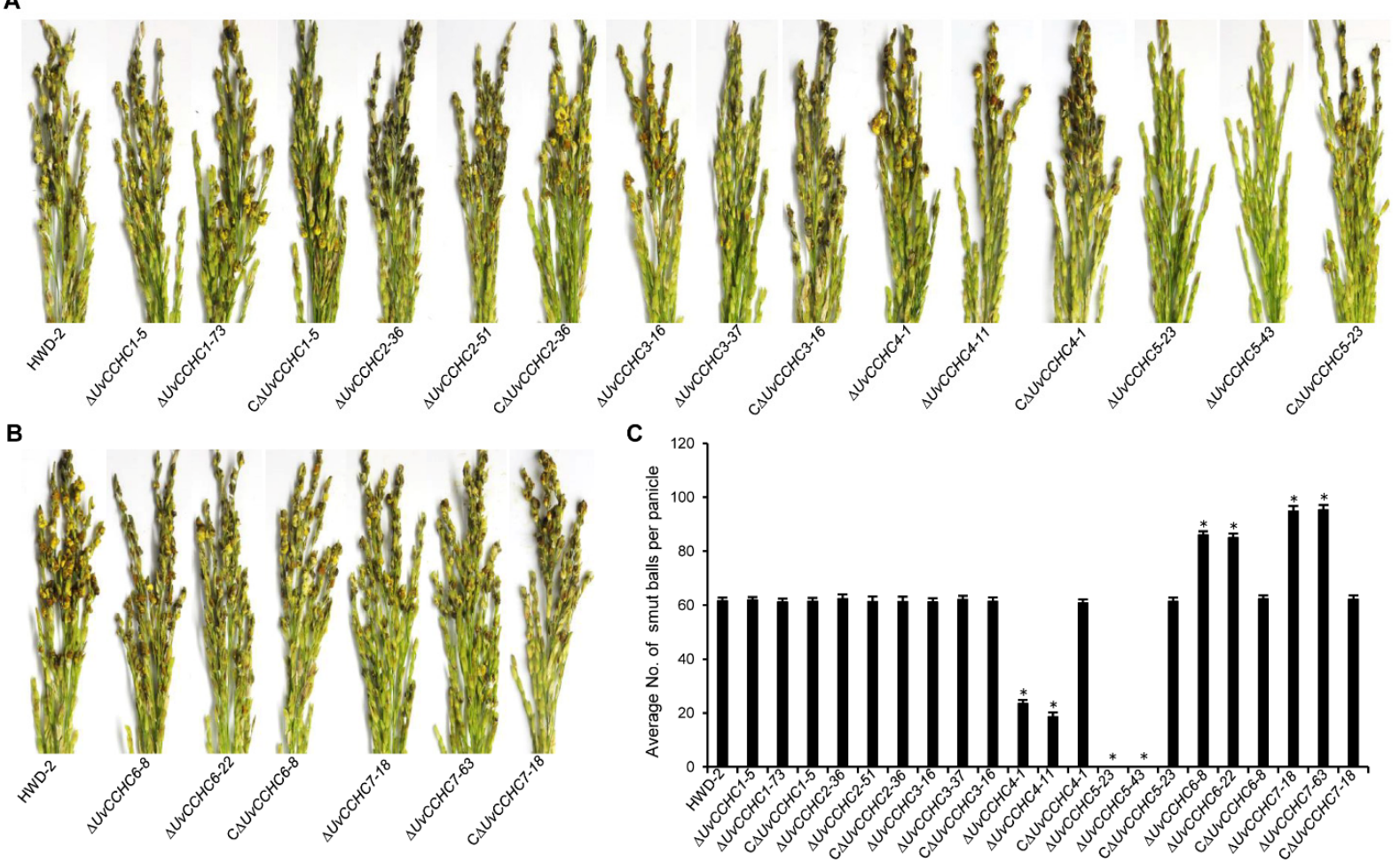

Figure 5. $U v C C H C$ genes are important for the virulence of $U$. virens. (A,B) Virulence assays of HWD-2, the $\triangle U v C C H C$ mutants, and complementation strains on rice spikelets at 21 dpi. (C) Average number of smut balls per panicle. Asterisks represent significant differences relative to HWD-2, as determined by LSD test at $p=0.05$.

We previously demonstrated that the transcription factor UvCom1 plays a key role in governing smut ball formation [35]. Here, we investigated whether UvCCHC5 shares the same function as UvCom1. UvCom1 regulates the expression of genes related to transporter activity, transmembrane transport, carbohydrate transport, and metabolism. Therefore, we performed RT-qPCR to detect the expression levels of genes related to membrane transport and sugar transport in mycelia. We found that these genes were significantly downregulated in $\triangle U v C C H C 5-23$ (Figure $7 \mathrm{C}$ ). In rice spikelets at $11 \mathrm{~d}$ after $U$. virens infection, wild-type hyphae absorb large amounts of nutrients from rice to form smut balls. We therefore measured the expression levels of these genes at $11 \mathrm{dpi}$, finding that most genes were significantly downregulated in $\triangle U v C C H C 5-23$ relative to the wild type at this stage (Figure 7D). These results suggest that $U$. virens with a deletion of UvCCHC5 is unable to form smut balls since the fungus itself has problems transporting rice nutrients, preventing it from utilizing these nutrients to form smut balls.

\subsection{Genome-Wide Identification of Genes Regulated by UvCCHC4}

To investigate the regulatory mechanism of the transcription factor UvCCHC4, we performed RNA-seq analysis to compare the gene expression profiles of HWD-2 vs. the $\triangle U v C C H C 4-1$ mutant using infected spikelets at 3 dpi. Based on $\log _{2}(\Delta U v C C H C 4-1 / H W D-2)$ values $>$ two-fold change, 810 differentially expressed genes (DEGs) were identified, including 652 downregulated and 158 upregulated genes (Figure 8A,B; Supplementary Table S2). Many of the DEGs were enriched in the GO terms structural constituent of ribosome, translation, ribosome, nucleolus, and rRNA binding (Figure 8C). KEGG enrichment analysis revealed that many DEGs were enriched in the pathways of mitochondrial biogenesis, ribosome, transporters, and ribosome biogenesis (Figure $8 \mathrm{D}$ ). To confirm the gene expression patterns revealed by RNA-seq, we performed RT-qPCR analysis using ten selected genes. The expression pattern of each downregulated or upregulated gene was consistent 
with that in the RNA-seq data (Figure 8E). These results indicate that UvCCHC4 plays key roles in ribosome activity and translation.

A
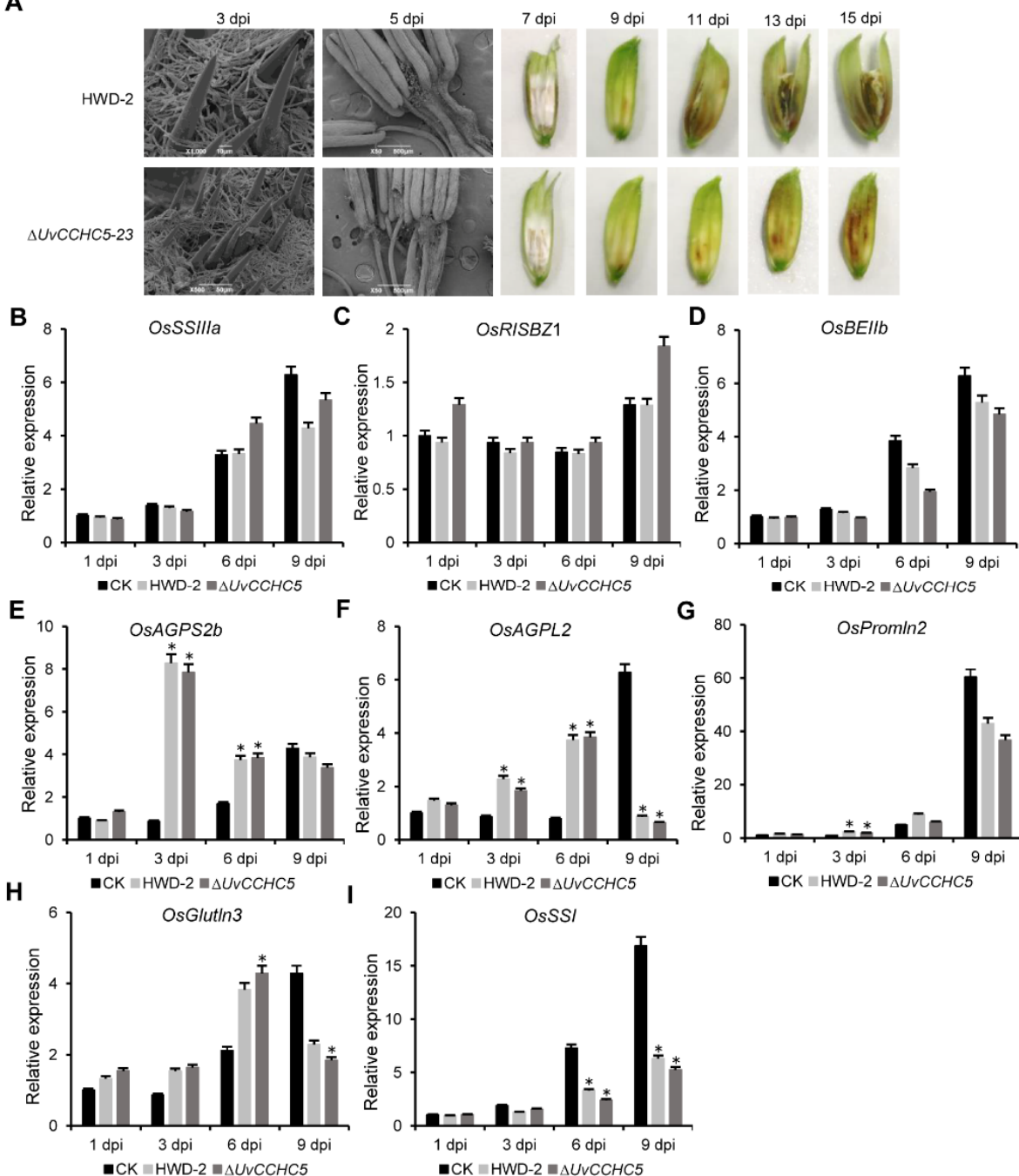

Figure 6. UvCCHC5 is a key transcription factor governing smut ball formation in rice. (A) Process of infection in inoculated rice spikelets. (B-I) Expression of genes associated with grain filling in rice at different stages after inoculation with $\triangle U v C C H C 5-23$ and HWD-2 $(1,3,6$, and $9 \mathrm{~d})$, as determined by RT-qPCR. Error bars represent the standard deviation of three replicates in independent experiments. Asterisks represent significant differences in comparison with CK at $P=0.05$. 
A

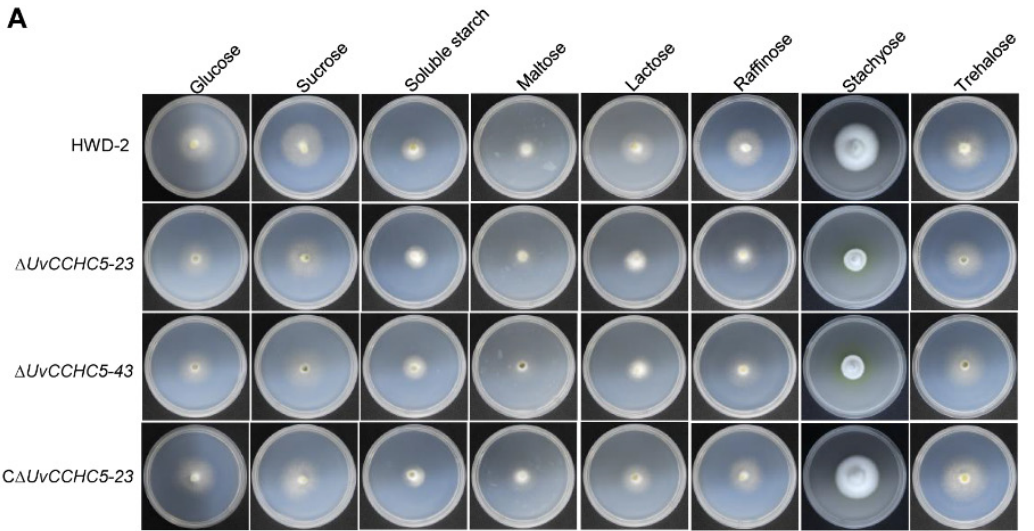

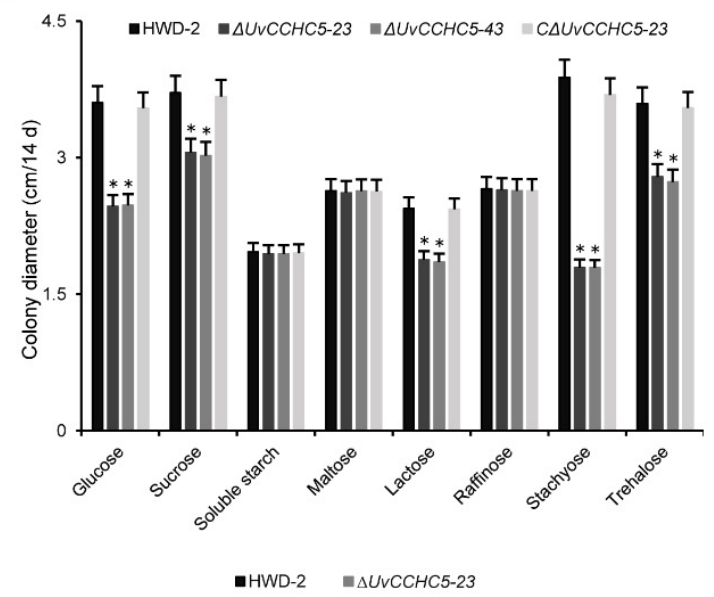

C

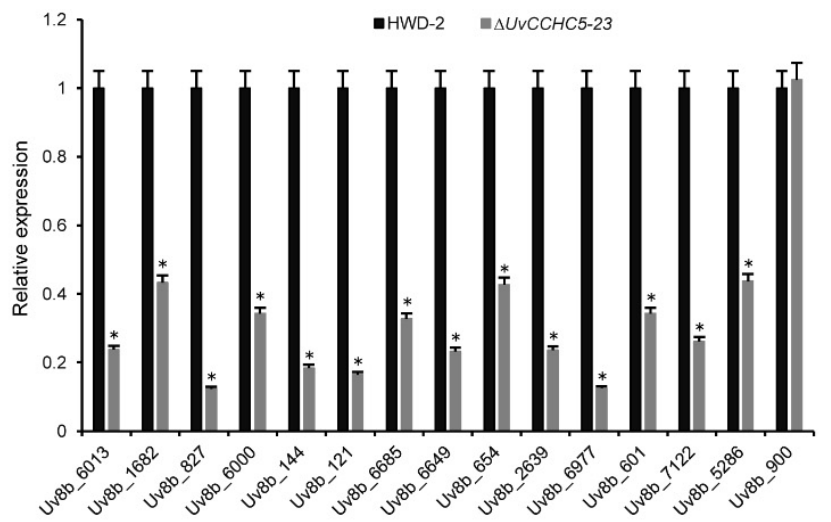

D

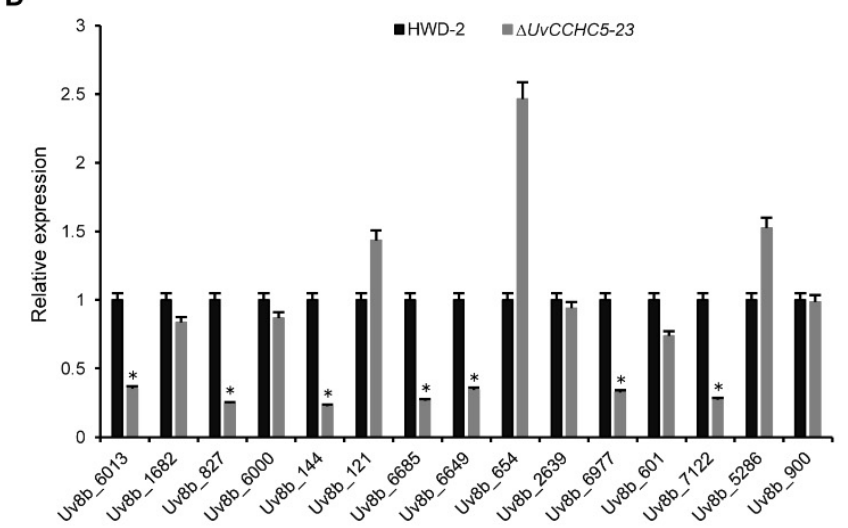

Figure 7. $\triangle U v C C H C 5$ mutants have defects in the utilization of different saccharides. (A) Colony morphology of $\triangle U v C C H C 5$ mutant and complementation strains at $14 \mathrm{~d}$ of culture on plates containing different saccharides. (B) Colony diameter of $\triangle U v C C H C 5$ mutants at $14 \mathrm{~d}$ of culture on plates containing different saccharides. (C,D) Results of RT-qPCR to validate the expression of 14 differentially expressed genes related to transmembrane transporters of $U$. virens in rice inoculated with HWD-2 and $\triangle U v C C H C 5-23$ in samples obtained at 0 (C) and 11 (D) dpi. Asterisks represent significant differences relative to HWD-2, as determined by LSD test at $p=0.05$.

\subsection{Subcellular Localization of UvCCHC4 and UvCCHC5 in U. virens}

Finally, to explore the subcellular localizations of UvCCHC4 and UvCCHC5, we inserted the pNeo3300III-GFP-UvCCHC4 and pNeo3300III-GFP-UvCCHC5 constructs into A. tumefaciens strain EHA105 and transformed conidia of $\triangle U v C C H C 4-1$ and $\triangle U v C C H C 5-23$ with these two vectors, respectively. Under confocal microscopy, the GFP signals in the transformed strains were observed in the nuclei of both conidia and vegetative hyphae (Figure 9A,B). Immunoblotting of these transformed strains using anti-GFP antibody showed a 101-kDa GFP-UvCCHC4 band and a 62-kDa GFP-UvCCHC5 band, indicating that the GFP-UvCCHC4 and GFP-UvCCHC5 fusion proteins were not completely cleaved (Figure 9C). These results indicate that $\mathrm{UvCCHC} 4$ and UvCCHC5 are localized to the nucleus in $U$. virens. 
A

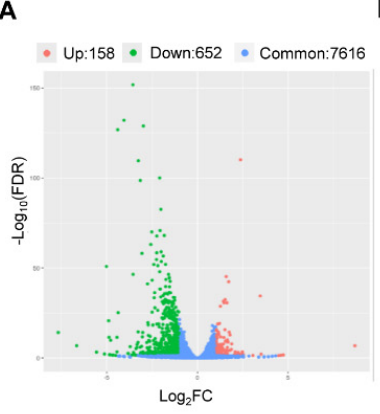

D

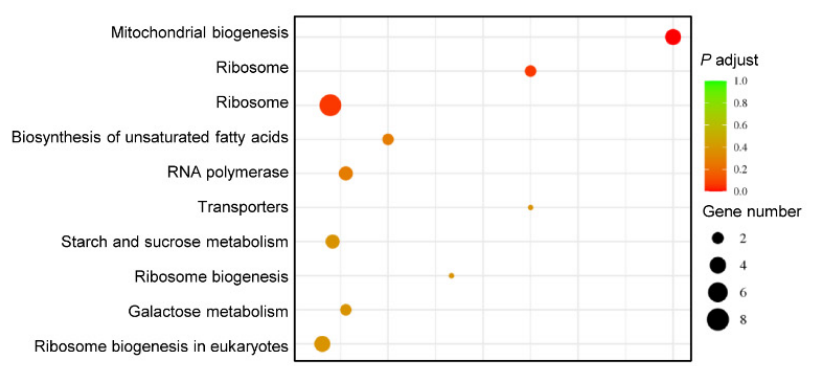

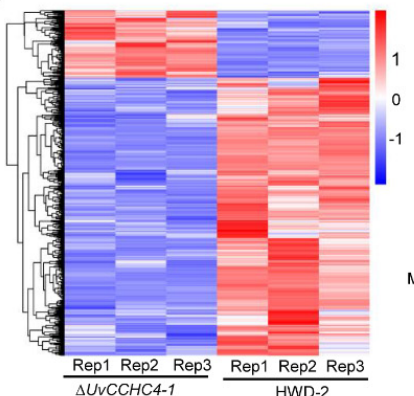
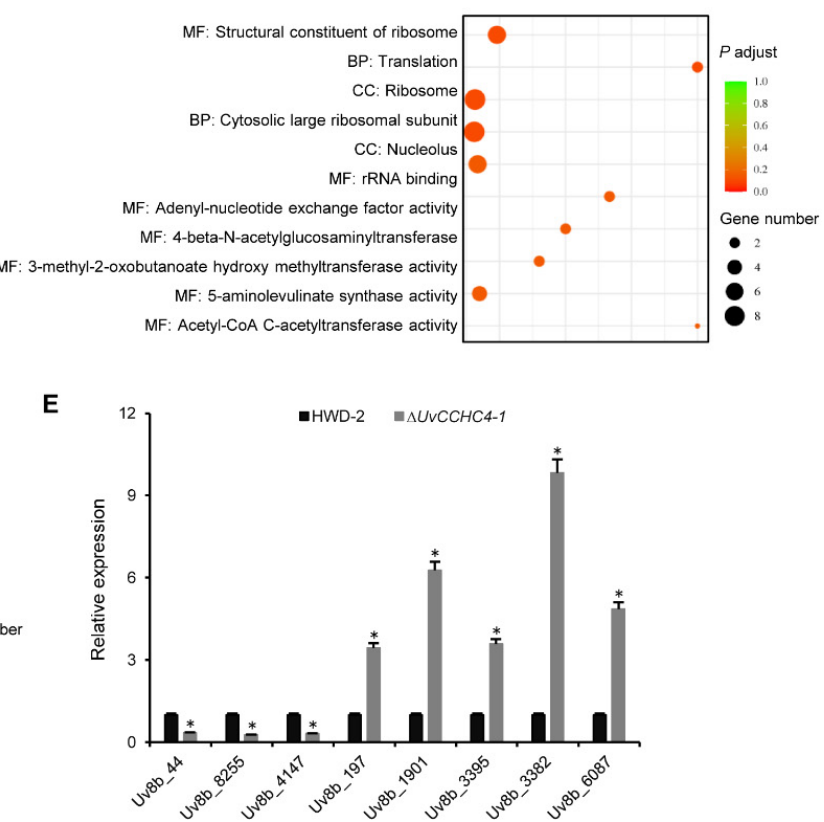

Figure 8. Transcriptomic analysis to identify DEGs in $\triangle U v C C H C 4-1$ vs. HWD-2. (A) Volcano plot of DEGs in $\triangle U v C C H C 4-1$ vs. HWD-2. (B) Heatmap of FRKM-normalized transcript levels of the 810 DEGs in each pair-wise comparison. (C) GO enrichment analysis of the DEGs. MF: Molecular function; BP: Biological process; CC: Cellular component. (D) KEGG enrichment analysis of the DEGs. (E) RT-qPCR to validate the DEGs. The relative transcript abundance of each gene from $\triangle U v C C H C 4-1$ was normalized by comparison with the gene from HWD-2 (relative transcript level =1). The asterisks represent significant differences between $\triangle U v C C H C 4-1$ and HWD-2 at $p=0.05$.

A

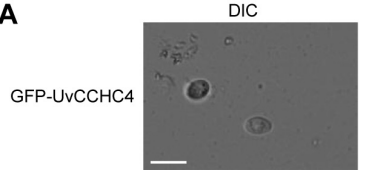

DIC

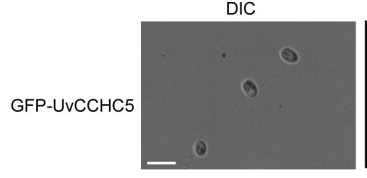

B

GFP-UvCCHC4

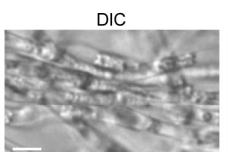

DIC

GFP-UvCCHC5

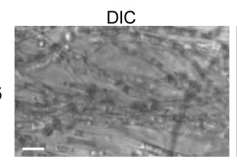

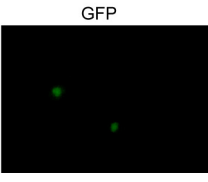

GFP

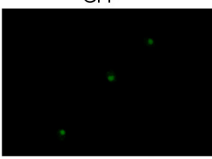

GFP

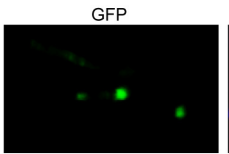

GFP

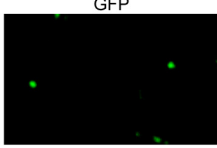

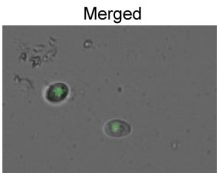

Merged

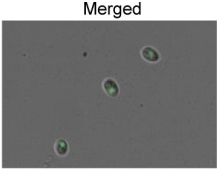

DAPI

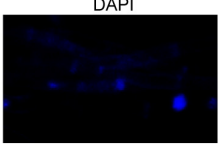

DAPI

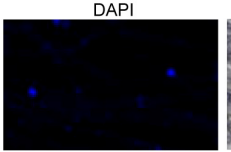

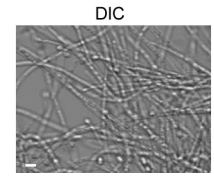

DIC

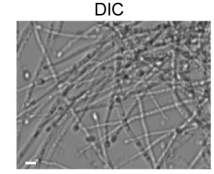

Merged

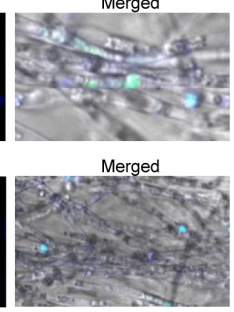

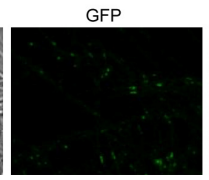

GFP
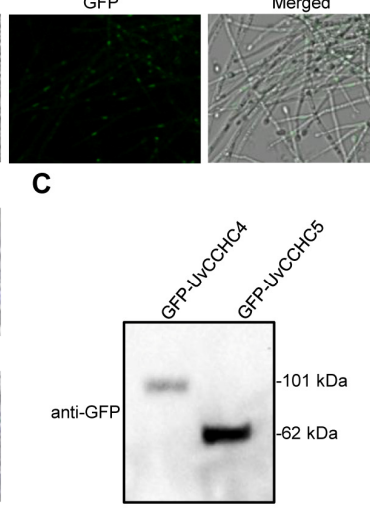

Figure 9. Subcellular localization of UvCCHC4 and UvCCHC5 in U. virens. (A) Subcellular localization of GFP-UvCCHC4 and GFP-UvCCHC5 in mycelia and conidia. DIC, differential interference contrast; GFP, green fluorescent protein; Scale bar $=10 \mu \mathrm{m}$. (B) Co-localization of DAPI and GFP-UvCCHC4 or GFP-UvCCHC5 in mycelia. DAPI, 4,6-diamidino-2phenylindole. Scale bar $=10 \mu \mathrm{m}$. (C) Immunoblot analysis examining GFP-UvCCHC4 and GFP-UvCCHC5 expression using an anti-GFP antibody. 


\section{Discussion}

Rice false smut, caused by $U$. virens, is one of the most serious rice diseases worldwide [28]. Many studies on $U$. virens have focused on its infection processes and strategies, whereas few have focused on genes involved in the virulence of $U$. virens. The identification of $U$. virens virulence factors has been hindered by its slow mycelial growth rate, its infection organ specificity, time- and labor-consuming artificial inoculation methods, and the relative difficulty of genetic transformation. Our understanding of the molecular mechanisms underlying $U$. virens virulence is quite limited. However, the efficiency of gene replacement in $U$. virens increased when the CRISPR (clustered regularly interspaced short palindromic repeat)/Cas9 system was utilized to generate gene-knockout strains [45]. This led to the functional identification of various pathogenic genes in $U$. virens, including iUvt3277, UvCom1, UvPRO1, UvAc1, UvPdeH, SCRE1, SCRE2 (UV_1261), UvBI-1, UvPmk1, UvCDC2, UvCdc3, UvCdc10, UvCdc11, UvCdc12, UvPal1, UvHox2, UvRpd3, UvSlt2, UvATG8, UvZnFTF1, UvMSN2, UvCGBP1, and UvEC1. These genes play important roles in the hyphal growth, conidiation, stress responses, and virulence of $U$. virens [46-55]. In the current study, we identified and functionally characterized CCHC-type zinc-finger proteins in $U$. virens. The CCHC-type zinc-finger protein homologs are well conserved in other filamentous ascomycetes, such as Magnaporthe oryzae and Fusarium graminearum. However, none of these homologs in fungi have been functionally characterized. In this study, we determined that the deletion of $\mathrm{UvCCHC}$ genes caused defects in the hyphal growth, conidiation, stress responses, and virulence of $U$. virens.

The $\triangle U v C C H C 5$ and $\triangle U v C o m 1$ mutants have the same infection phenotypes: Both mutants can infect the floral organs of rice, but the infected hyphae cannot expand to form smut balls. We propose that this defect is due to a problem in the nutrient supply chain established between the mutant fungus and rice. This defect renders the mutants incapable of utilizing large quantities of the nutrients produced by rice, preventing them from successfully forming smut balls. Previous studies have shown that deletion of UvCom1 does not affect the expression of genes related to rice filling, but the $\Delta U v \operatorname{Com} 1$ mutant has defects in transmembrane transport, transporter activity, carbohydrate transport, and metabolism [35]. Here we showed that the $\triangle U v C C H C 5$ and $\triangle U v C o m 1$ mutants induced the expression of rice-filling genes such as the wild type, whereas some transmembrane transport genes were significantly downregulated in the mutant fungi.

Biotrophic phytopathogenic fungi infect plants to obtain nutrients by redirecting carbon and nitrogen resources. Major facilitator superfamily (MFS) transporters target a wide range of substrates, including lipids, ions, amino acids and peptides, carbohydrates, and nucleosides [56]. Various genes related to MFS and transmembrane transport were significantly downregulated in mutant mycelia and during $U$. virens infection, including Uv8b_6013 (MFS multidrug transporter), Uv8b_6977 (Sugar transporter family protein), Uv8b_6685 (MFS multidrug transporter), Uv8b_144 (Carboxylic acid transport protein), Uv8b_6649 (MFS transporter), Uv8b_7122 (MFS sugar transporter), and Uv8b_827 (Oligopeptide transporter). UvCCHC5 and UvCom1 are two key transcription factors: further analysis of their activities could enrich our understanding of the formation of smut balls and the interaction between rice and $U$. virens. Whether the downstream target genes of the transcription factors UvCCHC 5 and UvCom 1 are the same, and whether they regulate the same pathway, will require further study.

We also performed RNA-seq to identify DEGs between HWD-2 and $\triangle U v C C H C 4-1$. Bioinformatics analysis revealed that several of these DEGs are enriched in the categories of structural constituent of ribosomes, translation, ribosome, nucleolus, rRNA binding, mitochondrial and ribosome biogenesis, and transporters. To further explore whether the genes regulated by $\mathrm{UvCCHC} 4$ are involved in the interaction between $U$. virens and rice, we searched the PHI-base database to identify DEGs regulated by $\mathrm{UvCCHC4}$, including genes encoding different types of transcription factors and transporters, G-protein-coupled receptors, protein kinases, and putative effectors. The results suggest that UvCCHC4 regulates diverse processes to mediate the interaction between $U$. virens and rice. Many 
DEGs encode secreted proteins (Supplementary Figure S3), including Uv8b_44,Uv8b_2253, Uv8b_2286,Uv8b_5436,Uv8b_5518, and Uv8b_6470, which were previously reported to induce cell death in Nicotiana benthamiana and rice [31,57]. We propose that UvCCHC4 helps regulate the expression of secreted proteins, which may themselves play important roles in the pathogenic process of $U$. virens. The roles of these secreted proteins in pathogenicity should be investigated in the future.

In summary, this is the first study to uncover the roles of CCHC-type zinc-finger proteins in regulating the development and virulence of a phytopathogen. Our findings shed light on the regulatory mechanism underlying the formation of smut balls.

\section{Conclusions}

In summary, our study revealed that UvCCHC genes affected the stress responses, vegetative growth, conidiation, and virulence of $U$. virens. We identified an important transcription factor, UvCCHC5, from this pathogen. UvCCHC5 was essential for the formation of rice smut balls; $\triangle U v C C H C 5$ mutants infected rice spikelets normally, but could not form smut balls, and lost the ability to stably utilize nutrients from the rice host. Sugar utilization experiments showed that the $\triangle U v C C H C 5$ mutants were defective in the utilization of glucose, sucrose, lactose, stachyose, and trehalose. $\triangle U v C C H C 5$ mutants induced the expression of rice-filling genes such as the wild type, whereas some transmembrane transport genes were significantly downregulated in the mutant fungi, and the resulting nutrient deficiencies prevent them from utilizing nutrients from rice to form smut balls. These findings improve our understanding of the molecular mechanism underlying the formation of smut balls in rice by $U$. virens.

Supplementary Materials: The following are available online at https: / www.mdpi.com/article / 10.3390/jof7110947/s1. Figure S1: Deletion and complementation of UvCCHC genes in U. virens. (A) Deletion and complementation strategies for $U v C C H C$ genes. (B) PCR verification of four $U v C$ CHC deletion mutants using primer pairs P1/P2, P3/P4, P3/P6 and P5/P6. (C) RT-PCR analysis of HWD-2 and $\triangle U v C C H C$ mutant and complementation strains; Figure S2: (A-G) Colony growth of $\triangle U v C C H C$ mutant strains on PSA supplied with $0.25 \mathrm{M} \mathrm{NaCl}, 0.5 \mathrm{M}$ sorbitol, $0.02 \%$ or $0.04 \%$ $\mathrm{H}_{2} \mathrm{O}_{2}, 0.03 \%$ SDS, $0.12 \mathrm{mg} / \mathrm{mL} \mathrm{CR}$, or $0.12 \mathrm{mg} / \mathrm{mL} \mathrm{CFW}$ after $14 \mathrm{~d}$ of incubation at $28^{\circ} \mathrm{C}$; Figure S3: Heatmap of the levels of secreted proteins in the $\triangle U C C H C 4-1$ mutant and HWD-2; Table S1: PCR primer pairs used in this study; Table S2: DEGs in rice spikelets infected with HWD-2 and $\triangle U v C C H C 4$ 1 at 3 dpi.

Author Contributions: X.C. (Xiaoyang Chen) and J.H. designed the experiments. Z.P. and X.C. (Xiaoyang Chen) performed most of the experiments and data analyses. H.L., C.L., L.P., Q.Q., L.Z., Y.D. and X.C. (Xiaolin Chen) provided technical support. X.C. (Xiaoyang Chen) wrote and revised the paper. All authors have read and agreed to the published version of the manuscript.

Funding: This work was supported by the National Natural Science Foundation of China (32072371). Institutional Review Board Statement: Not applicable.

Informed Consent Statement: Not applicable.

Acknowledgments: We are very grateful to Wenxian Sun (China Agricultural University) for providing the gene deletion vector $\mathrm{PGKO}$.

Conflicts of Interest: The authors declare no conflict of interest.

\section{References}

1. Coradetti, S.T.; Craig, J.P.; Xiong, Y.; Shock, T.; Tian, C.; Glass, N.L. Conserved and essential transcription factors for cellulase gene expression in ascomycete fungi. Proc. Natl. Acad. Sci. USA 2012, 109, 7397-7402. [CrossRef] [PubMed]

2. John, E.; Singh, K.B.; Oliver, R.P.; Tan, K.C. Transcription factor control of virulence in phytopathogenic fungi. Mol. Plant Pathol. 2021, 22, 858-881. [CrossRef]

3. Laity, J.H.; Lee, B.M.; Wright, P.E. Zinc finger proteins: New insights into structural and functional diversity. Curr. Opin. Struc. Biol. 2001, 11, 39-46. [CrossRef] 
4. Berg, J.M.; Shi, Y. The galvanization of biology: A growing appreciation for the roles of zinc. Science 1996, 271, $1081-1085$. [CrossRef]

5. Aceituno-Valenzuela, U.; Micol-Ponce, R.; Ponce, M.R. Genome-wide analysis of CCHC-type zinc finger (ZCCHC) proteins in yeast, Arabidopsis, and humans. Cell. Mol. Life Sci. 2020, 77, 3991-4014. [CrossRef]

6. Espinosa, J.M.; Portal, D.; Lobo, G.S.; Pereira, C.A.; Alonso, G.D.; Gómez, E.B.; Lan, G.H.; Pomar, R.V.R.; Flawiá, M.M.; Torres, H.N. Trypanosoma cruzi poly-zinc finger protein: A novel DNA/RNA-binding CCHC-zinc finger protein. Mol. Biochem. Parasit. 2003, 131, 35-44. [CrossRef]

7. Tan, Y.W.; Hong, W.; Liu, D.X. Binding of the 5'-untranslated region of coronavirus RNA to zinc finger CCHC-type and RNA-binding motif 1 enhances viral replication and transcription. Nucleic Acids Res. 2012, 40, 5065-5077. [CrossRef]

8. Luo, X.; Wang, B.; Gao, S.; Zhang, F.; Terzaghi, W.; Dai, M. Genome-wide association study dissects the genetic bases of salt tolerance in maize seedlings. J. Integr. Plant Biol. 2019, 61, 658-674. [CrossRef] [PubMed]

9. Wu, Q.; Wang, M.; Shen, J.; Chen, D.; Zheng, Y.; Zhang, W. ZmOST1 mediates abscisic acid regulation of guard cell ion channels and drought stress responses. J. Integr. Plant Biol. 2019, 61, 478-491. [CrossRef]

10. Sun, Y.; Li, J.Q.; Yan, J.Y.; Yuan, J.J.; Li, G.X.; Wu, Y.R.; Xu, J.M.; Huang, R.F.; Harberd, N.P.; Ding, Z.J. Ethylene promotes seed iron storage during Arabidopsis seed maturation via ERF95 transcription factor. J. Integr. Plant Biol. 2020, 62, 1193-1212. [CrossRef] [PubMed]

11. Liu, C.; Zhang, T.Z. Functional diversifications of GhERF1 duplicate genes after the formation of allotetraploid cotton. J. Integr. Plant Biol. 2019, 61, 60-74. [CrossRef]

12. Wang, W.; Wei, X.; Jiao, G.; Chen, W.; Wu, Y.; Sheng, Z.; Hu, S.; Xie, L.; Wang, J.; Tang, S.; et al. GBSS-BINDING PROTEIN, encoding a CBM48 domain-containing protein, affects rice quality and yield. J. Integr. Plant Biol. 2020, 62, 948-966. [CrossRef]

13. Tang, K.; Zhao, L.; Ren, Y.; Yang, S.; Zhu, J.K.; Zhao, C. The transcription factor ICE1 functions in cold stress response by binding to the promoters of CBF and COR genes. J. Integr. Plant Biol. 2020, 62, 258-263. [CrossRef]

14. Wang, L.; Gao, J.; Zhang, Z.; Liu, W.; Cheng, P.; Mu, W.; Su, T.; Chen, S.; Chen, F.; Jiang, J. Overexpression of CmSOS1 confers waterlogging tolerance in Chrysanthemum. J. Integr. Plant Biol. 2020, 62, 1059-1064. [CrossRef] [PubMed]

15. Radkova, M.; Revalska, M.; Kertikova, D.; Iantcheva, A. Zinc finger CCHC-type protein related with seed size in model legume species Medicago truncatula. Biotechnol. Biotech. Equip. 2019, 33, 278-285. [CrossRef]

16. Lu, X.; Zhou, Y.; Fan, F.; Peng, J.; Zhang, J. Coordination of light, circadian clock with temperature: The potential mechanisms regulating chilling tolerance in rice. J. Integr. Plant Biol. 2020, 62, 737-760. [CrossRef]

17. Zhao, M.; Wang, L.; Wang, J.; Jin, J.; Zhang, N.; Lei, L.; Gao, T.; Jing, T.; Zhang, S.; Wu, Y. Induction of priming by cold stress via inducible volatile cues in neighboring tea plants. J. Integr. Plant Biol. 2020, 62, 1461-1468. [CrossRef] [PubMed]

18. Zhang, C.; Lei, Y.; Lu, C.; Wang, L.; Wu, J. MYC2, MYC3, and MYC4 function additively in wounding-induced jasmonic acid biosynthesis and catabolism. J. Integr. Plant Biol. 2020, 62, 1159-1175. [CrossRef]

19. Liu, M.; Zhu, J.; Dong, Z. Immediate transcriptional responses of Arabidopsis leaves to heat shock. J. Integr. Plant Biol. 2020, 63, 468-483. [CrossRef]

20. Wang, Y.; Yu, Y.; Pang, Y.; Yu, H.; Zhang, W.; Zhao, X.; Yu, J. The distinct roles of zinc finger CCHC-type (ZCCHC) superfamily proteins in the regulation of RNA metabolism. RNA Biol. 2021, 1-20. [CrossRef]

21. de Paula, Q.A.; Mangrum, J.B.; Farrell, N.P. Zinc finger proteins as templates for metal ion exchange: Substitution effects on the C-finger of HIV nucleocapsid NCp7 using M (chelate) species (M=Pt, Pd, Au). J. Inorg. Biochem. 2009, 103, 1347-1354. [CrossRef]

22. Shimizu, K.; Chen, W.; Ashique, A.M.; Moroi, R.; Li, Y.P. Molecular cloning, developmental expression, promoter analysis and functional characterization of the mouse CNBP gene. Gene 2003, 307, 51-62. [CrossRef]

23. Berglund, J.A.; Chua, K.; Abovich, N.; Reed, R.; Rosbash, M. The splicing factor BBP interacts specifically with the pre-mRNA branchpoint sequence UACUAA C. Cell 1997, 89, 781-787. [CrossRef]

24. Jones, M.H.; Frank, D.N.; Guthrie, C. Characterization and functional ordering of Slu7p and Prp17p during the second step of pre-mRNA splicing in yeast. Proc. Natl. Acad. Sci. USA 1995, 92, 9687-9691. [CrossRef]

25. Vo, L.T.; Minet, M.; Schmitter, J.M.; Lacroute, F.; Wyers, F. Mpe1, a zinc knuckle protein, is an essential component of yeast cleavage and polyadenylation factor required for the cleavage and polyadenylation of mRNA. Mol. Cell. Biol. 2001, 21, 8346-8356. [CrossRef] [PubMed]

26. Sammons, M.A.; Samir, P.; Link, A.J. Saccharomyces cerevisiae Gis2 interacts with the translation machinery and is orthogonal to myotonic dystrophy type 2 protein ZNF9. Biochem. Biophys. Res. Commun. 2011, 406, 13-19. [CrossRef]

27. Miller, R.K.; D'Silva, S.; Moore, J.K.; Goodson, H.V. The CLIP-170 orthologue Bik1p and positioning the mitotic spindle in yeast. Curr. Top. Dev. Biol. 2006, 76, 49-87.

28. Sun, W.X.; Fan, J.; Fang, A.F.; Li, Y.J.; Tariqjaveed, M.; Li, D.Y.; Hu, D.W.; Wang, W.M. Ustilaginoidea virens: Insights into an emerging rice pathogen. Annu. Rev. Phytopathol. 2020, 58, 363-385. [CrossRef]

29. Koiso, Y.; Li, Y.; Iwasaki, S.; Kenji, H.; Tomowo, K.; Ryoichi, S.; Yoshikai, F.; Hiroshi, Y.; Zenji, S. Ustiloxins, antimitotic cyclic peptides from smut balls on rice panicles caused by Ustilaginoidea virens. J. Antibiot. 1994, 47, 765-773. [CrossRef] [PubMed]

30. Miyazaki, S.; Matsumoto, Y.; Uchihara, T.; Morimoto, K. High-performance liquid chromatographic determination of ustiloxin A in forage rice silage. J. Vet. Med. Sci. 2009, 71, 239-241. [CrossRef] 
31. Zhang, Y.; Zhang, K.; Fang, A.F.; Han, Y.Q.; Yang, J.; Xue, M.; Bao, J.; Hu, D.; Zhou, B.; Sun, X.; et al. Specific adaptation of Ustilaginoidea virens in occupying host florets revealed by comparative and functional genomics. Nat. Commun. 2014, 5, 3849. [CrossRef] [PubMed]

32. Chen, X.Y.; Liu, H.; Chen, X.L.; Huang, J.B.; Hsiang, T.; Zheng, L. ATAC-seq data for genome-wide profiling of transcription factor binding sites in the rice false smut fungus Ustilaginoidea virens. Mol. Plant Microbe Interact. 2021, 34, 830-834. [CrossRef]

33. Lv, B.; Zheng, L.; Liu, H.; Tang, J.T.; Hsiang, T.; Huang, J.B. Use of random T-DNA mutagenesis in identification of gene UvPRO1, a regulator of conidiation, stress response, and virulence in Ustilaginoidea virens. Front. Microbiol. 2016, 7, 2086. [CrossRef]

34. Yu, J.J.; Yu, M.N.; Song, T.Q.; Cao, H.J.; Yong, M.L.; Qi, Z.Q.; Du, Y.; Zhang, R.S.; Yin, X.L.; Liu, Y.F. A homeobox transcription factor UvHOX2 regulates chlamydospore formation, conidiogenesis and pathogenicity in Ustilaginoidea virens. Front. Microbiol. 2019, 10, 1071. [CrossRef]

35. Chen, X.Y.; Hai, D.; Tang, J.T.; Liu, H.; Huang, J.B.; Luo, C.X.; Hsiang, T.; Zheng, L. UvCom1 is an important regulator required for development and infection in the rice false smut fungus Ustilaginoidea virens. Phytopathology 2020, 110, 483-493. [CrossRef]

36. Song, T.Q.; Zhang, X.; Zhang, Y.; Liang, D.; Yan, J.; Yu, J.; Yu, M.; Cao, H.; Yong, M.; Pan, X.; et al. Genome-Wide Identification of Zn (2)-Cys (6) Class Fungal-Specific Transcription Factors (ZnFTFs) and Functional Analysis of UvZnFTF1 in Ustilaginoidea virens. Rice Sci. 2021, 28, 567-578.

37. Xu, Y.; Wu, S.; Yu, Z.; Moeketsi, E.K.; Yang, Z.; Zhang, Z.; Zhang, H. Transcription factor UvMsn2 is important for vegetative growth, conidiogenesis, stress response, mitochondrial morphology and pathogenicity in the rice false smut fungus Ustilaginoidea virens. Phytopathol. Res. 2021, 3, 16. [CrossRef]

38. Chen, X.; Li, P.; Liu, H.; Chen, X.; Huang, J.; Luo, C.; Li, G.; Hsiang, T.; Collinge, D.; Zheng, L. A novel transcription factor UvCGBP1 regulates development and virulence of rice false smut fungus Ustilaginoidea virens. Virulence 2021, 12, 1563-1579. [CrossRef] [PubMed]

39. Chen, X.Y.; Tang, J.T.; Pei, Z.X.; Liu, H.; Huang, J.B.; Luo, C.X.; Tom, H.; Zheng, L. The "pears and lemons" protein UvPal1 regulates development and virulence of Ustilaginoidea virens. Environ. Microbiol. 2020, 22, 5414-5432. [CrossRef] [PubMed]

40. Chen, X.Y.; Xu, Q.T.; Duan, Y.H.; Liu, H.; Chen, X.L.; Huang, J.B.; Luo, C.X.; Zhou, D.X.; Zheng, L. Ustilaginoidea virens modulates lysine 2-hydroxyisobutyrylation in rice flowers during infection. J. Integr. Plant Biol. 2021, 63, 1801-1814. [CrossRef] [PubMed]

41. Li, B.; Dewey, C.N. RSEM: Accurate transcript quantification from RNA-Seq data with or without a reference genome. BMC Bioinform. 2011, 12, 323. [CrossRef]

42. Xie, C.; Mao, X.; Huang, J.; Ding, Y.; Wu, J.; Dong, S. KOBAS 2.0: A web server for annotation and identification of enriched pathways and diseases. Nucleic Acids Res. 2011, 39, 316-322. [CrossRef]

43. Fan, J.; Guo, X.Y.; Li, L.; Huang, F.; Sun, W.X.; Li, Y.; Huang, Y.Y.; Xu, Y.J.; Shi, J.; Lei, Y.; et al. Infection of Ustilaginoidea virens intercepts rice seed formation but activates grain-filling-related genes. J. Integr. Plant. Biol. 2015, 57, 577-590. [CrossRef]

44. Song, J.H.; Wei, W.; Lv, B.; Lin, Y.; Yin, W.X.; Peng, Y.L.; Schnabel, G.; Huang, J.B.; Jiang, D.H.; Luo, C.X. Rice false smut fungus hijacks the rice nutrients supply by blocking and mimicking the fertilization of rice ovary. Environ. Microbiol. 2016, 18, 3840-3849. [CrossRef]

45. Liang, Y.; Han, Y.; Wang, C.; Jiang, C.; Xu, J.R. Targeted deletion of the USTA and UvSLT2 genes efficiently in Ustilaginoidea virens with the CRISPR-Cas9 system. Front. Plant Sci. 2018, 9, 699. [CrossRef] [PubMed]

46. Chen, X.Y.; Pei, Z.X.; Li, P.P.; Li, X.B.; Duan, Y.H.; Liu, H.; Chen, X.L.; Zheng, L.; Luo, C.X.; Huang, J.B. Quantitative proteomics analysis reveals the function of the putative ester cyclase UvEC1 in the pathogenicity of the rice false smut fungus Ustilaginoidea virens. Int. J. Mol. Sci. 2021, 22, 4069. [CrossRef] [PubMed]

47. Chen, X.Y.; Li, X.B.; Li, P.P.; Chen, X.L.; Liu, H.; Huang, J.B.; Luo, C.X.; Tom, H.; Zheng, L. Comprehensive identification of lysine 2-hydroxyisobutyrylated proteins in Ustilaginoidea virens reveals the involvement of lysine 2-hydroxyisobutyrylation in fungal virulence. J. Integr. Plant. Biol. 2021, 63, 409-425. [CrossRef] [PubMed]

48. Zheng, M.T.; Ding, H.; Huang, L.; Wang, Y.H.; Yu, M.N.; Zheng, R.; Yu, J.J.; Liu, Y.F. Low-affinity iron transport protein Uvt3277 is important for pathogenesis in the rice false smut fungus Ustilaginoidea virens. Curr. Genet. 2017, 63, 131-144. [CrossRef]

49. Fang, A.F.; Gao, H.; Zhang, N.; Zheng, X.H.; Qiu, S.S.; Li, Y.J.; Zhou, S.; Cui, F.H.; Sun, W.X. A novel effector gene SCRE2 contributes to full virulence of Ustilaginoidea virens to rice. Front. Microbiol. 2019, 10, 845. [CrossRef]

50. Xie, S.L.; Wang, Y.F.; Wei, W.; Li, C.Y.; Liu, Y.; Qu, J.S.; Meng, Q.H.; Lin, Y.; Yin, W.X.; Yang, Y.N.; et al. The Bax inhibitor UvBI-1, a negative regulator of mycelial growth and conidiation, mediates stress response and is critical for pathogenicity of the rice false smut fungus Ustilaginoidea virens. Curr. Genet. 2019, 65, 1-13. [CrossRef]

51. Guo, W.W.; Gao, Y.X.; Yu, Z.M.; Xiao, Y.H.; Zhang, Z.G.; Zhang, H.F. The adenylate cyclase UvAc1 and phosphodiesterase UvPdeH control the intracellular cAMP level, development, and pathogenicity of the rice false smut fungus Ustilaginoidea virens. Fungal Genet. Biol. 2019, 129, 65-73. [CrossRef] [PubMed]

52. Tang, J.T.; Bai, J.; Chen, X.Y.; Zheng, L.; Liu, H.; Huang, J.B. Two protein kinases UvPmk1 and UvCDC2 with significant functions in conidiation, stress response and pathogenicity of rice false smut fungus Ustilaginoidea virens. Curr. Genet. 2019, 66, 409-420. [CrossRef]

53. Meng, S.; Xiong, M.; Jagernath, J.S.; Wang, C.; Qiu, J.; Shi, H.; Kou, Y. UvAtg8-mediated autophagy regulates fungal growth, stress responses, conidiation, and pathogenesis in Ustilaginoidea virens. Rice 2020, 13, 56. [CrossRef] [PubMed]

54. Zhang, N.; Yang, J.; Fang, A.F.; Wang, J.Y.; Li, D.Y.; Li, Y.J.; Wang, S.Z.; Cui, F.H.; Yu, J.J.; Liu, Y.F.; et al. The essential effector SCRE1 in Ustilaginoidea virens suppresses rice immunity via a small peptide region. Mol. Plant Pathol. 2020, 21, 445-459. [CrossRef] 
55. Fan, J.; Du, N.; Li, L.; Li, G.; Wang, Y.; Zhou, Y.; Hu, X.; Liu, J.; Zhao, J.; Li, Y.; et al. A core effector UV_1261 promotes Ustilaginoidea virens infection via spatiotemporally suppressing plant defense. Phytopathol. Res. 2019, 1, 11. [CrossRef]

56. Yan, N. Structural advances for the major facilitator superfamily (MFS) transporters. Trends Biochem. Sci. 2013, 38, 151-159. [CrossRef] [PubMed]

57. Fan, J.; Yang, J.; Wang, Y.Q.; Li, G.B.; Li, Y.; Huang, F.; Wang, W.M. Current understanding on Villosiclava virens, a unique flower-infecting fungus causing rice false smut disease. Mol. Plant Pathol. 2016, 17, 1321-1330. [CrossRef] 